\title{
Finite-Time Behavior of Inner Systems
}

\author{
Jobert H. A. Ludlage, Member, IEEE, Siep Weiland, Anton A. Stoorvogel, Senior Member, IEEE, and \\ Ton A. C. P. M. Backx
}

\begin{abstract}
In this paper, we investigate how nonminimum phase characteristics of a dynamical system affect its controllability and tracking properties. For the class of linear time-invariant dynamical systems, these characteristics are determined by transmission zeros of the inner factor of the system transfer function. The relation between nonminimum phase zeros and Hankel singular values of inner systems is studied and it is shown how the singular value structure of a suitably defined operator provides relevant insight about system invertibility and achievable tracking performance. The results are used to solve various tracking problems both on finite as well as on infinite time horizons. A typical receding horizon control scheme is considered and new conditions are derived to guarantee stabilizability of a receding horizon controller.
\end{abstract}

Index Terms-Control systems, optimal control, stability, statespace methods, tracking.

\section{INTRODUCTION}

$\mathbf{I}$ $\mathrm{N}$ THE beginning of the development of control theory, it was recognized [7], [21], [42], [56] that the process behavior limits the achievable performance of the controlled system irrespective of which control strategy is applied. Ziegler and Nichols [56] had already observed that for a "miserably designed process" even the finest controller may not deliver the desired performance. Hence, already in the process design phase it is important to have a thorough understanding of how a process unit will limit the achievable performance of the controlled unit [2], [11], [31], [43], [45]. Obviously, such an understanding is also important for controller design [7], [29], [33], [35], [42], [46], [56]. A detailed analysis of the relation between the open-loop process dynamics and the requirements posed on the controlled process during operation, is indispensable to make choices between different and often conflicting control objectives. Because of this, there are various motivations for the present paper.

First, most modern control design techniques are based on the optimization of criterion functions in which weighting parameters reflect the desired behavior. Linear quadratic control, $H_{\infty}$ optimal control and optimization of control inputs over finite time laps are typical examples of design strategies which involve

Manuscript received February 22, 2002; revised October 8, 2002 and February 26, 2003. Recommended by Associate Editor F. Callier.

J. H. A. Ludlage and T. A. C. P. M. Backx are with IPCOS Technology Inc., 5282 WV Boxtel, The Netherlands.

S. Weiland is with the Department of Electrical Engineering, Eindhoven University of Technology, 5600 MB Eindhoven, The Netherlands (e-mail: s.weiland@tue.nl).

A. A. Stoorvogel is with the Department of Mathematics and Computing Science, Eindhoven University of Technology, 5600 MB Eindhoven, The Netherlands. He is also with the Department of Information Technology and Systems, Delft University of Technology, Delft, The Netherlands.

Digital Object Identifier 10.1109/TAC.2003.814108 an appropriate choice of weighting parameters. The better the fundamental process limitations are understood, the fewer trial and error iterations for the selection of these parameters will be necessary.

Second, classical control strategies (such as PID controllers) have found widespread applications in process industry, but have the inherent shortcoming to ignore multivariable phenomena. In general, the sensitivity of process variables in specific input and output directions is not analyzed prior to the design of these controllers. Such an analysis is indispensable to improve process performance.

Third, this research has been motivated by the need to improve model predictive control techniques. Model predictive control is probably the most widely used multivariable control design technique in industry. In refinery and petrochemical industries, these controllers have become a standard tool for unit optimization and they receive an ever growing interest in other applications. Commercially available model predictive controllers are all based on finite time horizon optimizations. Unlike their growing interest, questions related to tracking and guaranteed stability of such controlled systems are only partly understood and investigated.

In the literature, a number of definitions appeared to formalize the influence of open-loop dynamics on closed loop behavior of processes. Typical notions include (input-output) controllability [42], [46], [56], plant capacity [21], reproducibility [7], dynamic operability [2], and dynamic resilience [33], [45]. Most of these definitions are not very precise, but they do express how the process puts limitations on achievable closed-loop behavior. Nevertheless, these concepts do not express to what extent the process behavior limits the desired controlled behavior, i.e., the control specifications are not taken into account in the assessment of issues related to process controllability.

It is well known that nonminimum phase plants are more difficult to control and that nonminimum phase zeros impose intrinsic limitations on stability, performance and robustness. Bode's pioneering work on the sensitivity integral [5] and Freudenberg-Looze's Poisson integral expressions [14], [15] quantify in an analytic way that for nonminimum phase systems the magnitude of the sensitivity function $|S|$ can be made less than one over some frequencies only if it is larger than one over a complementary range of frequencies. Therefore, desirable properties of the sensitivity function in one frequency range have to be traded off against undesirable behavior at other frequencies: the so called "analytic tradeoff" [6], [9], [12], [14], [15].

For nonminimum phase systems, the aforementioned integral relations provide insight in the sensitivity properties of the controlled system in relatively simple cases. Indeed, these relations 
allow to analyze the effect of one or at most a few dominant nonminimum phase zeros, usually for single-input-single-output systems only. In more complex cases, such insight is usually absent. A second, and frequently applied, approach to consider the influence of nonminimum phase zeros on closed-loop behavior is to standardize the problem. In most cases, this means that an inner-outer factorization is applied on the transfer function of the plant and the complementary sensitivity is chosen equal to the inner factor [19], [20], [46]. The influence of nonminimum phase zeros on closed-loop performance is then reflected by the frequency response of the resulting sensitivity matrix. A second type of standardization is analog to the Wolovich and Falb interactor matrix [55] and amounts to choosing the sensitivity matrix $S\left(e^{j \omega}\right)$ triangular [34], [51], [52]. In this approach, it is assumed that the outputs are arranged in descending order of importance: output one has absolute priority above output two, and so on. As a result, the influence of nonminimum phase zeros on the outputs of the closed-loop system is manifest only in the outputs of lowest priority. In many cases, such an approach results in undesirable behavior at the process input and at the lowest priority outputs. From an analysis point of view, a major disadvantage of standardization procedures is that the freedom in the control design is not entirely used but, instead, partly fixed.

In contrast to the aforementioned approaches, this paper aims to provide a multivariable structural analysis of input-output properties that limit controllability, invertibility, tracking and stabilizability properties of a plant. We investigate the singular value structure of Toeplitz-like operators associated with the inner factor of a plant and appropriate time intervals. A key advantage of this approach is that the time intervals which are relevant for tracking, control or estimation are explicitly taken into account. For arbitrary time horizons (finite as well as infinite), it is shown that the singular vectors of these operators span signals which can not be tracked or signals which are difficult to track. The relation between these singular values and nonminimum phase zeros is explained and the results of the analysis are applied to a number of relevant control problems. Throughout this paper we will focus on inner systems. This may seem restrictive at first sight, but is well motivated for at least two reasons. First, since the zero structure and invertibility properties of a linear time-invariant plant are encoded in the inner factor of the transfer function, it is reasonable to focus the analysis on the inner factors only. Second, the graph of any linear time-invariant plant (i.e., the set of all $\mathcal{L}_{2}$ bounded input-output pairs which are compatible with the plant) admits a representation as the image (or kernel) of an inner (or co-inner) transfer function and, as such, defines an inner (or co-inner) representation for any linear time-invariant plant.

The paper is organized as follows. Preliminary definitions and results are given in Section II. Section III deals with the formal problem statement. Main theoretical results are presented in Section IV. In Section V, we discuss how these results can be applied to two tracking problems. Section VI treats an important stabilization problem in receding horizon control and derives a parametrization of end-point weights that result in a stabilizing MPC controller. Some simulation results are presented in Section VII. Conclusions are collected in Section VIII. The paper has been written such that readers interested in the control applications can read Sections $\mathrm{V}$ and VI independent of the preceding theory.

\section{PReliminary Definitions AND Notation}

Define for $T \subseteq \mathbb{Z}$ and $q \geq 1$ the function class

$$
\ell_{2}\left(T, \mathbb{R}^{q}\right):=\left\{x:\left.T \rightarrow \mathbb{R}^{q}\left|\|x\|_{\ell_{2}(T)}^{2}:=\sum_{t \in T}\right| x(t)\right|^{2}<\infty\right\}
$$

where $|x(t)|$ is the standard Euclidean norm of the vector $x(t)$. We write $\|\cdot\|_{2}$ for $\|\cdot\|_{\ell_{2}(T)}$ whenever the signal domain $T$ is clear from the context. $\ell_{2}\left(T, \mathbb{R}^{q}\right)$ is equipped with the standard inner product $\langle x, y\rangle:=\sum_{t \in T} y^{\top}(t) x(t)$. We abbreviate $\ell_{2}\left(T, \mathbb{R}^{q}\right)$ to $\ell_{2}$ or $\ell_{2}(T)$ whenever the usage is clear from the context. Let $\mathbb{Z}_{-}$and $\mathbb{Z}_{+}$be the negative and the nonnegative elements of $\mathbb{Z}$, respectively. The support of a signal $x$, defined on $T$, is $\operatorname{supp}(x):=\{t \in T \mid x(t) \neq 0\}$. The shift $s$ maps $T$ to $s T:=\{t+1 \mid t \in T\}$. Mostly, $T$ will be an interval, that is, $T$ will be the set of all integers $t \in \mathbb{Z}$ with $a<t<b$ where $a, b \in \mathbb{Z} \cup\{\infty,-\infty\}$. An interval is called finite if $a, b \in \mathbb{Z}$, it is infinite if either $a=-\infty$ or $b=\infty$. It is empty if $a \geq b$.

\section{A. Poles and Zeros}

Let $R H_{\infty}^{p \times m}$ be the class of all $p \times m$ stable proper rational transfer functions (all poles strictly inside the unit disc). A transfer function $G \in R H_{\infty}^{p \times m}$ will be identified with the state-space system

$$
\begin{aligned}
x(t+1) & =A x(t)+B u(t) \\
y(t) & =C x(t)+D u(t)
\end{aligned}
$$

if, and only if, $G(z)=C(z I-A)^{-1} B+D,|\lambda(A)|<1$ (i.e., $A$ is stable) and the quadruple $(A, B, C, D)$ is minimal. In that case, we will say that $(A, B, C, D)$ realizes $G \in R H_{\infty}$. Let $G(z)=Y(z) \Sigma(z) U(z)$ be the Smith-McMillan decomposition of $G$ where $U(z)$ and $Y(z)$ are unimodular polynomial matrices, $\Sigma(z)=\operatorname{diag}\left(\Sigma^{\prime}(z), 0\right)$ and $\Sigma^{\prime}$ is a full-rank matrix of the form [23]

$$
\Sigma^{\prime}(z)=\operatorname{diag}\left(\epsilon_{1}(z) / \psi_{1}(z), \ldots, \epsilon_{r}(z) / \psi_{r}(z)\right)
$$

where the polynomials $\varepsilon_{i}$ and $\psi_{i}$ are coprime, $\epsilon_{i+1}$ divides $\epsilon_{i}$ and the polynomial $\psi_{i}$ divides $\psi_{i+1}$ for $i=1, \ldots, r-1$, with $r$ the normal rank of $G$. A complex number $\lambda$ is a pole of $G$ if it is a root of the polynomial $\psi_{r}(z)$, it is a transmission zero of $G$ if it is a root of the polynomial $\epsilon_{1}(z)$. We call $\lambda$ a nonminimum phase zero if it is a transmission zero with $|\lambda| \geq 1$, it is called a minimum phase zero if it is a transmission zero with $|\lambda|<1$.

\section{B. The Gramians}

Let $T_{u}$ and $T_{y}$ be two intervals. The controllability gramian and the observability gramian associated with (1) are given by

$$
\begin{aligned}
P\left(T_{u}\right) & :=\sum_{t \in s T_{u}} A^{-t} B B^{\top}\left(A^{\top}\right)^{-t} \\
Q\left(T_{y}\right) & :=\sum_{t \in T_{y}}\left(A^{\top}\right)^{t} C^{\top} C A^{t} .
\end{aligned}
$$


The (possibly infinite) controllability and observability matrices are

$$
\begin{aligned}
& \mathcal{C}\left(T_{u}\right):=\operatorname{row}\left(A^{-t} B, t \in s T_{u}\right) \\
& \mathcal{O}\left(T_{y}\right):=\operatorname{col}\left(C A^{t}, t \in T_{y}\right)
\end{aligned}
$$

respectively, where "row" means placing subsequent entries side by side (lowest indexed entry on left) and "col" means stacking subsequent entries (lowest indexed entry on top). If $A$ is stable (i.e., $|\lambda(A)|<1$ ), the gramians are well defined if either $T_{u} \subseteq \mathbb{Z}_{-}$and $T_{y} \subseteq \mathbb{Z}_{+}$, or $A$ is invertible and $T_{y}$ is left-bounded and $T_{u}$ is right-bounded. Whenever the gramians are well defined $P\left(T_{u}\right)=\mathcal{C}\left(T_{u}\right) \mathcal{C}\left(T_{u}\right)^{\top}$ and $Q\left(T_{y}\right)=\mathcal{O}\left(T_{y}\right)^{\top} \mathcal{O}\left(T_{y}\right)$. If $N>0$ and $T_{u}=[-N,-1]$, $T_{y}=[0, N-1]$, then $P\left(T_{u}\right), Q\left(T_{y}\right), \mathcal{C}\left(T_{u}\right)$ and $\mathcal{O}\left(T_{y}\right)$ are abbreviated as $P_{N}, Q_{N}, \mathcal{C}_{N}$ and $\mathcal{O}_{N}$, respectively. To further simplify notation, we define $P:=P_{\infty}, Q:=Q_{\infty}, \mathcal{C}:=\mathcal{C}_{\infty}$ and $\mathcal{O}:=\mathcal{O}_{\infty}$. We call (1) input balanced, output balanced or input-output balanced [32] if both $P$ and $Q$ are diagonal and, $P=I, Q=I$ or $P=Q$, respectively. It is well known [32] that for any minimal stable state representation (1) there exists a convenient basis of the state space such that the system is balanced in either of these forms. The following lemma gives a system theoretic interpretation of the gramians.

Lemma II.1: Let (1) be stable, and suppose that $T_{u} \subseteq \mathbb{Z}-$ and $T_{y} \subseteq \mathbb{Z}_{+}$. Let $y_{0}: T_{y} \rightarrow \mathbb{R}^{p}$ denote the output of (1) resulting from input $u=0$ and initial condition $x(0)=x_{0}$. Then

1) $\left\|y_{0}\right\|_{2}^{2}=x_{0}^{\top} Q\left(T_{y}\right) x_{0}$;

2) if $P\left(T_{u}\right)$ is invertible then

$$
\begin{array}{r}
\inf \left\{\|u\|_{2}^{2} \mid u \in \ell_{2}, \operatorname{supp}(u) \subseteq T_{u}, x \in \ell_{2}, x(0)=x_{0}\right\} \\
=x_{0}^{\top} P\left(T_{u}\right)^{-1} x_{0} .
\end{array}
$$

\section{Proof:}

1) First observe that $|\lambda(A)|<1$ implies that $Q\left(T_{y}\right)$ is well defined for any finite or infinite interval $T_{y} \subseteq \mathbb{Z}_{+}$. Since $y_{0}(t)=C A^{t} x_{0}$, it is immediate that

$$
\left\|y_{0}\right\|_{2}^{2}=\sum_{t \in T_{y}} x_{0}^{\top}\left(A^{\top}\right)^{t} C^{\top} C A^{t} x_{0}=x_{0}^{\top} Q\left(T_{y}\right) x_{0} .
$$

2) Suppose $T_{u} \subseteq \mathbb{Z}$ - and define $T_{u}^{\prime}:=\left\{t \in T_{u}, t \geq t_{0}\right\}$ with $t_{0} \in T_{u}$ fixed such that $P\left(T_{u}^{\prime}\right)$ is invertible. (Since $P\left(T_{u}\right)$ is invertible such a $t_{0}$ obviously exists). For all $t \geq t_{0}$, we have that

$$
x(t)=A^{t-t_{0}} x\left(t_{0}\right)+\sum_{k=t_{0}}^{t-1} A^{t-k-1} B u(k) .
$$

Let $x\left(t_{0}\right)=0$. Using (3), a control $u \in \ell_{2}$ with $\operatorname{supp}(u) \subseteq T_{u}^{\prime}$ steers the state $x$ from 0 at time $t_{0}$ to $x_{0}$ at time 0 if and only if: $x_{0}=\sum_{k \in T_{u}^{\prime}} A^{-k-1} B u(k)=$ $\mathcal{C}\left(T_{u}^{\prime}\right) \operatorname{col}\left(u(k), k \in T_{u}^{\prime}\right)$. Hence, for all $x_{0} \in \mathbb{R}^{n}$ there exists such a $u$ because $P\left(T_{u}^{\prime}\right)$ invertible implies that $\operatorname{rank} \mathcal{C}\left(T_{u}^{\prime}\right)=n$, in which case the optimal control in (2) with $T_{u}$ replaced by $T_{u}^{\prime}$ [optimal in the sense of having a minimal norm $\left.\|u\|_{\ell_{2}\left(T_{\nu}^{\prime}\right)}\right]$ is given by $u^{*}(t)=0$, $t \notin T_{u}^{\prime}$ and $\operatorname{col}\left(u^{*}(t), t \in T_{u}^{\prime}\right):=\mathcal{C}\left(T_{u}^{\prime}\right)^{\top} P\left(T_{u}^{\prime}\right)^{-1} x_{0}$.
$\left[P\left(T_{u}^{\prime}\right)\right.$ is invertible since $\left.\operatorname{rank} \mathcal{C}\left(T_{u}^{\prime}\right)=n\right]$. Consequently, $\left\|u^{*}\right\|_{\ell_{2}\left(T^{\prime}\right)}^{2}=x_{0}^{\top} P\left(T_{u}^{\prime}\right)^{-1} x_{0}$. Finally, observe that $T_{u}^{\prime} \subseteq T_{u} \Longrightarrow P\left(T_{u}^{\prime}\right) \leq P\left(T_{u}\right)$. Consequently

$$
\begin{aligned}
\inf \left\{\|u\|_{\ell_{2}\left(T_{u}\right)}^{2} \mid u\right. & \left.\in \ell_{2}, \operatorname{supp}(u) \subseteq T_{u}, x \in \ell_{2}, x(0)=x_{0}\right\} \\
& =\inf _{T_{u}^{\prime} \subseteq T_{u}} x_{0}^{\top} P\left(T_{u}^{\prime}\right)^{-1} x_{0}=x_{0}^{\top} P\left(T_{u}\right)^{-1} x_{0} .
\end{aligned}
$$

This proves statement 2 ).

\section{Singular Values}

Let $T_{u}$ and $T_{y}$ be two intervals. We will be interested in the system (1) where the time instants of the outputs are restricted to $T_{y}$ and the inputs are assumed to have support in $T_{u}$. Precisely, we define an operator

$$
\Psi_{T_{u}, T_{y}}: \ell_{2}\left(T_{u}\right) \rightarrow \ell_{2}\left(T_{y}\right)
$$

such that $y=\Psi_{T_{u}, T_{y}} u$ is the output $y: T_{y} \rightarrow \mathbb{R}^{p}$ of the system (1) subject to the initial condition $\lim _{t \rightarrow-\infty} x(t)=0$, and the input signal $u \in \ell_{2}$ with $\operatorname{support} \operatorname{supp}(u) \subseteq T_{u}$. Note that this operator is defined in terms of the external input-output signals of (1). This means that any property of $\Psi_{T_{u}, T_{y}}$ is independent of the particular realization $(A, B, C, D)$ of the transfer function $G$ associated with (1).

Following standard terminology, $\Psi_{T_{u}, T_{y}}$ is called a Hankel operator if $\max T_{u}<\min T_{y}$, it is called a Toeplitz operator if $\min T_{u}=\min T_{y}$. Let

$$
\left\|\Psi_{T_{u}, T_{y}}\right\|:=\sup _{0 \neq u \in \ell_{2}\left(T_{u}\right)} \frac{\left\|\Psi_{T_{u}, T_{y}} u\right\|_{2}}{\|u\|_{2}}
$$

be its induced norm. We call this norm a Hankel norm if $\Psi_{T_{u}, T_{y}}$ is Hankel and a Toeplitz norm if $\Psi_{T_{u}, T_{y}}$ is Toeplitz. Furthermore, the singular values $\sigma_{i}\left(\Psi_{T_{u}, T_{y}}\right)$ are assumed to be ordered according to $\sigma_{1} \geq \sigma_{2} \geq \cdots$ and are called Hankel or Toeplitz singular values whenever $\Psi_{T_{u}, T_{y}}$ is Hankel or Toeplitz. The Hankel singular values associated with the transfer function $G \in R H_{\infty}$ are the Hankel singular values of $\Psi_{\mathbb{Z}_{-}, \mathbb{Z}_{+}}$.

Lemma II.2: If $T_{u} \subseteq \mathbb{Z}_{-}, T_{y} \subseteq \mathbb{Z}_{+}$, then $\Psi_{T_{u}, T_{y}}$ is Hankel and $\left\|\Psi_{T_{u}, T_{y}}\right\|=\lambda_{\max }^{1 / 2}\left(P\left(T_{u}\right) Q\left(T_{y}\right)\right)$. If $T_{u}=T_{y}=\mathbb{Z}_{+}$then $\Psi_{T_{u}, T_{y}}$ is Toeplitz and $\left\|\Psi_{T_{u}, T_{y}}\right\|=\|G\|_{\infty}$.

Proof: With $T_{u} \subseteq \mathbb{Z}_{-}$and $T_{y} \subseteq \mathbb{Z}_{+}, \Psi_{T_{u}, T_{y}}$ is Hankel by definition and $\Psi_{T_{u}, T_{y}}=\mathcal{O}\left(T_{y}\right) \mathcal{C}\left(T_{u}\right)$. Hence $\operatorname{rank}\left(\Psi_{T_{u}, T_{y}}\right) \leq$ $n$ and the $i$ th singular value $\sigma_{i}$ of $\Psi_{T_{u}, T_{y}}$ satisfies

$$
\sigma_{i}^{2}=\lambda_{i}\left[\mathcal{C}^{\top}\left(T_{u}\right) \mathcal{O}^{\top}\left(T_{y}\right) \mathcal{O}\left(T_{y}\right) \mathcal{C}\left(T_{u}\right)\right]=\lambda_{i}\left[P\left(T_{u}\right) Q\left(T_{y}\right)\right]
$$

which proves the first statement. The second statement is a standard application of Parseval's theorem and can be found in [13] and [53].

\section{Inner and Co-Inner Systems}

A real rational transfer function $G$ is called inner if $G \in$ $R H_{\infty}^{p \times m}$ and if it is norm preserving in the sense that $\|G u\|_{2}=$ $\|u\|_{2}$ for all $u \in L_{2}$. It is called co-inner if its conjugate $G^{*}$ is inner. A (co-)inner transfer function $G$ is called structural (co-)inner or minimum phase (co-)inner if it has no transmission zeros. A function $G \in H_{\infty}$ is called outer if the image of the multiplicative operator $G$ under $\mathrm{H}_{2}$ is a dense subset of $\mathrm{H}_{2}$. For 
proper rational transfer functions $G \in R H_{\infty}^{p \times p}$ this is equivalent to saying that $G$ has no zeros (i.e., constant rank) in the open unit disk.

The following result is a special case of the bounded real lemma (the "lossless" bounded real lemma) for discrete time systems; see, e.g., [1].

Lemma II.3: Suppose that $G \in R H_{\infty}^{p \times m}$ has a minimal realization $(A, B, C, D)$. Then $G$ is inner if and only if there exists $Q>0$ such that

$$
\left(\begin{array}{ll}
A & B \\
C & D
\end{array}\right)^{\top}\left(\begin{array}{ll}
Q & 0 \\
0 & I
\end{array}\right)\left(\begin{array}{ll}
A & B \\
C & D
\end{array}\right)=\left(\begin{array}{ll}
Q & 0 \\
0 & I
\end{array}\right) .
$$

Moreover, in that case $p \geq m$ and all Hankel singular values of $G$ are smaller or equal to one. If $p=m$ then all Hankel singular values of $G$ equal one.

A similar result holds for co-inner transfer functions in which case (4) reads

$$
\left(\begin{array}{ll}
A & B \\
C & D
\end{array}\right)\left(\begin{array}{ll}
P & 0 \\
0 & I
\end{array}\right)\left(\begin{array}{ll}
A & B \\
C & D
\end{array}\right)^{\top}=\left(\begin{array}{ll}
P & 0 \\
0 & I
\end{array}\right) .
$$

In fact, under the given conditions the matrices $Q$ in (4) and $P$ in (5) are uniquely defined and coincide with the gramians $Q$ and $P$, respectively, defined in Section II-B. The notation is therefore consistent. We call the state representation (1) isometric or co-isometric whenever the corresponding transfer function $G(z)=C(I z-A)^{-1} B+D$ is inner or co-inner, respectively.

It is well known [53] that every full rank transfer function $G \in R H_{\infty}^{p \times m}$, admits either an inner-outer factorization $G=$ $G_{\mathrm{i}} G_{\mathrm{o}}$ with $G_{\mathrm{i}} \in R H_{\infty}^{p \times m}$ inner and $G_{\mathrm{o}} \in R H_{\infty}^{m \times m}$ outer or an outer-co-inner factorization $G=G_{\mathrm{o}} G_{\mathrm{i}}$ with $G_{\mathrm{i}} \in R H_{\infty}^{p \times m}$ co-inner and $G_{\mathrm{o}} \in R H_{\infty}^{p \times p}$ outer (depending on whether $p \geq$ $m$ or $p \leq m$ ). For any such factorization, the nonminimum phase zeros of $G$ and the nonminimum phase zeros of the (co)inner factor $G_{\mathrm{i}}$ coincide. Actually, the number of transmission zeros of a (co)-inner system is directly related to the number of Hankel singular values which are equal to one. Specifically, the following holds.

Lemma II.4: All transmission zeros of a (co-)inner system are nonminimum phase zeros. The transmission zeros are a subset of the inverse of the eigenvalues of $A$ and the number of zeros equals the number of Hankel singular values equal to one.

Proof: See [29], [47], and [50].

We infer from Lemma II.4 that the study of the "nonminimum phase behavior" (encoded in $G_{\mathrm{i}}$ ) of a system $G$ can be separated from the study of its "gain behavior" (encoded in $G_{\mathrm{o}}$ ). Innerouter factorizations can be used to reformulate a large class of feedback control problems as optimization problems which are affine in an unconstrained parameter (the Youla parameter) that ranges over (classes of) $H_{\infty}$ functions. See, e.g., [35], [46], and [53]. In particular, this shows that sensitivity minimization is limited by the inner part of the system.

\section{PROBLEM STATEMENTS}

We will investigate the following problems.

Definition III.1 (General Problem Formulation): Let $T_{u}$ and $T_{y}$ be two time intervals in $\mathbb{Z}$ and let $T_{u y}=T_{u} \cup T_{y}$. Given an inner system $G \in R H_{\infty}^{p \times m}$ and a reference signal $y_{\text {ref }} \in \ell_{2}$, consider the following questions.

1) Find a control $u$ with support $\operatorname{supp}(u) \subseteq T_{u}$, such that the output $y:=\Psi_{T_{u}, T_{u y}} u$ tracks $y_{\text {ref }}$ on $T_{u}$ in that $y(t)=$ $y_{\text {ref }}(t)$ for all $t \in T_{u}$.

2) If such a control exists, characterize the norms $\|u\|_{\ell_{2}\left(T_{u}\right)}$ and $\|y\|_{\ell_{2}\left(T_{y}\right)}$ as function of the length of the time interval $T_{u}$ and the reference signal.

3) If such a control does not exists, relax the tracking requirement to achieve that $y(t)=y_{\text {ref }}(t)$ for all $t \in T_{u}^{\prime}$ with $T_{u}^{\prime}$ a strict subset of $T_{u}$ and characterize $\|u\|_{\ell_{2}\left(T_{u}\right)}$ and $\|y\|_{\ell_{2}\left(T_{y}\right)}$ as function of the time interval $T_{u}$ and the reference signal.

4) Find a control $u$ with support $\operatorname{supp}(u) \subseteq T_{u}$, that minimizes the quadratic criterion $\left.|| y\right|_{T_{u}}-\left.y_{\text {ref }}\right|_{T_{u}} \|^{2}+x_{\mathrm{e}}^{\top} E x_{\mathrm{e}}$ and investigate for which end-point weights $E$ a receding horizon implementation of such a control will yield an exponentially stable controlled system. Here, $x_{\mathrm{e}}$ denotes the end-point state of the optimization interval.

In words, in the first three problems we are interested in the question how much control energy is needed to achieve tracking on the interval $T_{u}$ (or a proper subset of $T_{u}$ ) and to what extend tracking on this interval affects the future behavior of the output. The last problem involves the stability of a receding horizon scheme. Note that these problems are of evident interest for applications in model predictive control (MPC) where norm-constrained input and output signals are analyzed on finite time horizons and where stability of systems controlled by receding horizon controllers is of eminent importance.

In view of the definition of the operator $\Psi_{T_{u}, T_{y}}$, the problems 1,2 , and 3 are in fact equivalent to the question of existence and construction of the inverse of the Toeplitz operator $\Psi_{T_{u}, T_{u}}$. In the next section, we will therefore study the invertibility of this operator, viewed as function of the length of $T_{u}$. Problem 4 will be resolved in Section VI and its solution will, to some extend, be independent of the solution of problems 1 , 2 , and 3 .

The focus on inner systems may seem restrictive at first sight, but is justified and motivated for at least two reasons. First, in order to analyze how nonminimum phase zeros of a plant restrict controlled behavior, we infer from Section II-D, that the inner (or co-inner) factor of the transfer function of the plant reflects the nonminimum phase zero structure of the system. To simplify the analysis we therefore focus on the inner factor only. Second, if $P$ is an arbitrary real rational transfer function of a discrete time, linear time-invariant plant, with $\bar{u}$ its $\bar{m}$ dimensional input and $\bar{y}$ its $\bar{p}$-dimensional output, then we may identify such a plant with its graph

$$
\operatorname{graph}(P):=\left\{\operatorname{col}(\bar{y}, \bar{u}) \in L_{2} \mid \bar{y}=P \bar{u}\right\}
$$

Hence, $\operatorname{graph}(P)$ consists of the collection of all possible $L_{2}$-bounded input-output pairs which are compatible with the plant. The graph is well defined for both stable and unstable systems and it is an application of the Beurling-Lax theorem (for details, see [41]) that $\operatorname{graph}(P)$ can be represented as either the image (or null space) of an inner (or co-inner) multiplicative operator acting on $L_{2}$. Precisely, $P$ admits a 
normalized right- and normalized left-coprime factorization ${ }^{1}$ $P=N D^{-1}=\tilde{D}^{-1} \tilde{N}$ such that $G:=\operatorname{col}(N, D)$ is inner, $\tilde{G}:=\operatorname{row}(-\tilde{D}, \tilde{N})$ is co-inner and

$$
\begin{aligned}
\operatorname{graph} P & =\operatorname{im} G=\left\{y=\operatorname{col}(\bar{y}, \bar{u}) \mid y=G u, u \in L_{2}\right\} \\
& =\operatorname{ker} \tilde{G}=\left\{u=\operatorname{col}(\bar{y}, \bar{u}) \mid 0=\tilde{G} u, u \in L_{2}\right\}
\end{aligned}
$$

where $G \in R H_{\infty}^{p \times m}$ and $\tilde{G} \in R H_{\infty}^{\bar{p} \times p}$ with $p=\bar{m}+\bar{p}$ and $m=$ $\bar{m}$. See [17], [38], and [53]. In words, the set of $L_{2}$ bounded input-output pairs which are compatible with the plant can be represented as the image or kernel of an inner or co-inner function. Consequently, graph $(P)$ (or, for that matter, $P$ ) admits an inner and a co-inner representation. The general problem formulated in Definition III.1 is therefore relevant for tracking problems which involve the graph of arbitrary (linear time-invariant) plants. With the graph of a plant represented as the image of an inner transfer function, the reference signal $y_{\text {ref }} \in \ell_{2}$ in Definition III. 1 assumes the form $y_{\text {ref }}=\operatorname{col}\left(\bar{y}_{\text {ref }}, \bar{u}_{\text {ref }}\right)$ and therefore defines a reference signal for both the input as well as the output of the plant $P$. Likewise, the control signal $u$ in Definition III.1 becomes a new (auxiliary) signal which is related to the actual input $\bar{u}$ of the plant by setting $\bar{u}=\operatorname{row}\left(0, I_{\bar{m}}\right) G u$. With this interpretation, the general problem formulated in Definition III.1 defines a tracking problem on finite horizons in the joint input-output variables of the plant.

\section{BeHAVIOR OF InNER Systems on Finite TIME}

In this section, the structural properties of the Toeplitz operator $\Psi_{T_{u}, T_{u}}$ associated with an inner transfer function $G$ will be studied. Let $T_{u}$ be an arbitrary finite interval of $\mathbb{Z}$ and let $T_{y}:=\left\{t \in \mathbb{Z} \mid t>\max \left(T_{u}\right)\right\}$. Then, for all $u \in \ell_{2}$ with $\operatorname{supp}(u) \subseteq T_{u}$, we have

$$
\|u\|_{2}^{2}=\left\|\Psi_{T_{u}, T_{u}} u\right\|_{2}^{2}+\left\|\Psi_{T_{u}, T_{y}} u\right\|_{2}^{2}
$$

Hence, $\Psi_{T_{u}, T_{u}}^{\top} \Psi_{T_{u}, T_{u}}=I-\Psi_{T_{u}, T_{y}}^{\top} \Psi_{T_{u}, T_{y}}$. In the next theorem, this observation is used to characterize the singular values of the Toeplitz operator $\Psi_{T_{u}, T_{u}}$.

Theorem VI.1: Let $G \in R H_{\infty}^{p \times m}$ be inner with minimal realization $(A, B, C, D)$ and McMillan degree $n$. Let $T_{u}$ be a finite interval of length $N$, let $T_{y}:=\left\{t \in \mathbb{Z} \mid t>\max \left(T_{u}\right)\right\}$ and suppose that $N \geq n$. Then, the following hold.

1) $m N-n$ singular values of $\Psi_{T_{u}, T_{u}}$ equal one.

2) The $n$ smallest singular values of $\Psi_{T_{u}, T_{u}}$ equal the square root of the nonzero eigenvalues of $I-\Psi_{T_{u}, T_{y}}^{\top} \Psi_{T_{u}, T_{y}}$ and are a nonincreasing function of the length $N$ of $T_{u}$. For these singular values we have

$$
\begin{aligned}
\lim _{N \rightarrow \infty} \sigma_{m N-n+i}\left(\Psi_{T_{u}, T_{u}}\right) & =\lim _{N \rightarrow \infty}\left(1-\sigma_{n+1-i}^{2}\left(\Psi_{T_{u}, T_{y}}\right)\right)^{1 / 2} \\
& =\left(1-\sigma_{n+1-i}^{2}\left(\Psi_{\mathbb{Z}_{-}, \mathbb{Z}_{+}}\right)\right)^{1 / 2}
\end{aligned}
$$

where $i=1, \ldots, n$.

\footnotetext{
${ }^{1}$ That is, there exist $N, D, \tilde{N}$ and $\tilde{D}$ all belonging to $R H_{\infty}$, with $D$ and $\tilde{D}$ square and nonsingular as rational matrices, $G:=\operatorname{col}(N, D)$ and $\tilde{G}:=$ row $(-\tilde{D}, \tilde{N})$ inner with a left inverse in $R H_{\infty}$ and co-inner with a right-inverse in $R H_{\infty}$, respectively, such that $P=N D^{-1}=\tilde{D}^{-1} \tilde{N}$. The invertibility conditions mean that the Bezout equations $X N+Y D=I$ and $\tilde{N} \tilde{X}+$ $\tilde{D} \tilde{Y}=I$ are solvable over $R H_{\infty}$ or, equivalently, that the pairs $(N, D)$ and $(\tilde{N}, \tilde{D})$ are right-, respectively, left coprime over $R H_{\infty}$. We refer to [53] for explicit constructions of such factorizations.
}

3) The span of the right singular vectors of $\Psi_{T_{u}, T_{u}}$ corresponding to its smallest $n$ singular values equals $\operatorname{imC}_{N}^{\top}$.

4) The span of the left singular vectors of $\Psi_{T_{u}, T_{u}}$ corresponding to its smallest $n$ singular values contains the span of

$-\mathcal{O}_{N} P\left(A^{\top}\right)^{N_{t}} \operatorname{col}\left(\left(C P A^{\top}+D B^{\top}\right)\left(A^{\top}\right)^{-t-1}, t \in[-N,-1]\right)$.

Proof: Let $\Psi_{T_{u}, T_{u}}=Y \Sigma U^{T}$ be a singular value decomposition of $\Psi_{T_{u}, T_{u}}$. Since $G$ is inner, we have that $p \geq m$ (Lemma II.3) and

$$
I-\Psi_{T_{u}, T_{y}}^{\top} \Psi_{T_{u}, T_{y}}=\Psi_{T_{u}, T_{u}}^{\top} \Psi_{T_{u}, T_{u}}=U \Sigma^{\top} \Sigma U^{\top} .
$$

The $m N$ entries on the diagonal of $\Sigma^{\top} \Sigma$ coincide with the eigenvalues of $I-\Psi_{T_{u}, T_{y}}^{\top} \Psi_{T_{u}, T_{y}}$. Since $\operatorname{rank}\left(\Psi_{T_{u}, T_{y}}\right)=\operatorname{rank} \mathcal{O}\left(T_{y}\right) \mathcal{C}\left(T_{u}\right)=n$ whenever $N \geq n$, we infer that $\operatorname{rank}\left(I-\Sigma^{\top} \Sigma\right)=n$, i.e., $m N-n$ singular values of $\Psi_{T_{u}, T_{u}}$ are one. This proves statement 1.

Because

$$
I-\Sigma^{\top} \Sigma=U^{\top} \Psi_{T_{u}, T_{y}}^{\top} \Psi_{T_{u}, T_{y}} U \geq 0
$$

the other $n$ singular values of $\Psi_{T_{u}, T_{u}}$ are strictly smaller than 1. Since $T_{u}$ has length $N$, there exists an integer $k$ such that $T_{u}^{\prime}:=s^{k} T_{u}=[-N,-1]$. Define $T_{y}^{\prime}:=s^{k} T_{y}=\mathbb{Z}_{+}$. Since the system is time invariant we infer from Lemma II.2 that the singular values $\sigma_{i}\left(\Psi_{T_{u}, T_{y}}\right)=\sigma_{i}\left(\Psi_{T_{u}^{\prime}, T_{y}^{\prime}}\right)=\lambda_{i}^{1 / 2}\left(P\left(T_{u}^{\prime}\right) Q\left(T_{y}^{\prime}\right)\right)$. These numbers are nondecreasing functions of $N$ as $T_{y}^{\prime}$ does not depend on $N$ and $P\left(T_{u}^{\prime}\right) \leq P\left(T_{u}^{\prime \prime}\right)$ whenever $T_{u}^{\prime} \subseteq T_{u}^{\prime \prime} \subseteq \mathbb{Z}-$. Using (8), this implies that the nonone singular values of $\Psi_{T_{u}, T_{u}}$ are nonincreasing functions of $N$. More specifically, if $u_{j}$ is the $j$ th column of $U$ and $\sigma_{j}$ the $j$ th singular value of $\Psi_{T_{u}, T_{u}}$, then for $j=1, \ldots, m N$

$$
\begin{aligned}
u_{j}^{\top} \Psi_{T_{u}, T_{y}}^{\top} \Psi_{T_{u}, T_{y}} u_{j} & =u_{j}^{\top}\left(I-U \Sigma^{\top} \Sigma U^{\top}\right) u_{j} \\
& =u_{j}^{\top}\left(1-\sigma_{j}^{2}\right) u_{j}
\end{aligned}
$$

which shows that the nonzero singular values of $\Psi_{T_{u}, T_{y}}$ are given by $\left(1-\sigma_{j}^{2}\right)^{1 / 2}$ where $m N-n<j \leq m N$. Thus, for $i=1, \ldots, n$

$$
\sigma_{m N-n+i}\left(\Psi_{T_{u}, T_{u}}\right)=\left(1-\sigma_{n+1-i}^{2}\left(\Psi_{T_{u}, T_{y}}\right)\right)^{1 / 2}
$$

and since these are nonincreasing functions of $N$ their limits (as $N \rightarrow \infty$ ) exist and we obtain (6). This proves statement 2).

To prove statement 3$)$, define $\mathcal{U}:=\operatorname{span}\left\{u_{j} \mid m N-n<\right.$ $j \leq m N\}$ and infer from (9), the time invariance of the system and Lemma II.2 that

$$
u_{j}^{\top}\left(1-\sigma_{j}^{2}\right) u_{j}=u_{j}^{\top} \mathcal{C}_{N}^{\top} Q \mathcal{C}_{N} u_{j}
$$

For $j \leq m N-n$ this expression is zero. Since $Q$ is invertible, this shows that $\mathcal{U} \subseteq \operatorname{imC}_{N}^{\top}$. On the other hand, $n=\operatorname{dim} \mathcal{U} \geq$ $\operatorname{dim} \mathcal{C}_{N}$ and, hence, we must have that $\mathcal{U}=\operatorname{imC}_{N}^{\top}$.

Let $\mathcal{Y}$ be the span of the left singular vectors of $\Psi_{T_{u}, T_{u}}$ corresponding to its $n$ smallest singular values. Then, obviously, $\Psi_{T_{u}, T_{u}} \mathcal{U} \subseteq \mathcal{Y}$ and $\Psi_{T_{u}^{\prime}, T_{u}^{\prime}} \mathcal{U} \subseteq \mathcal{Y}$. Let $y \in \Psi_{T_{u}^{\prime}, T_{u}^{\prime}} \mathcal{U}$. As 
$\mathcal{U}=\operatorname{im\mathcal {C}_{N}^{\top }}$, we can write $y=\Psi_{T_{u}^{\prime}, T_{u}^{\prime}} \mathcal{C}_{N}^{\top} x$ for some $x \in \mathbb{R}^{n}$. Using (1), we obtain that

$$
y(t)=C A^{N+t} x(-N)+\left[C P_{N+t}\left(A^{\top}\right)^{-t}+D B^{\top}\left(A^{\top}\right)^{-t-1}\right] x
$$

with $t \in[-N,-1]$ define the components of $y$ in that $y=$ $\operatorname{col}(y(t), t \in[-N,-1])$. Let $x(-N)=0$ and substitute in (10) the identity $P_{N+t}=P-A^{N+t} P\left(A^{\top}\right)^{N+t}$ with $P$ the controllability gramian. Then range over all $x \in \mathbb{R}^{n}$ to obtain (7). This proves statement 4).

From Theorem IV.1 we infer that the closer to one are the singular values of the Hankel operator $\Psi_{T_{u}, T_{y}}$, the closer to zero are the singular values of the Toeplitz $\Psi_{T_{u}, T_{u}}$ and, hence, the closer $\Psi_{T_{u}, T_{u}}$ gets to singularity. Moreover, by Lemma II.4 there is a one-one correspondence between nonminimum phase zeros and unit Hankel singular values. This observation has important consequences for control. Indeed, if a reference signal $y_{\mathrm{ref}}: T_{u} \rightarrow \mathbb{R}^{p}$ needs to be tracked by choosing a suitable control $u: T_{u} \rightarrow \mathbb{R}^{m}$, then

$$
u=\Psi_{T_{u}, T_{u}}^{\dagger} y_{\mathrm{ref}}
$$

with $\dagger$ denoting the Moore-Penrose inverse, defines the control with minimal $\ell_{2}$ norm such that the output $y=\Psi_{T_{u}, T_{u}} u$ optimally approximates $y_{\text {ref }}$ in the $\ell_{2}$ sense. By Theorem IV.1, the norm $\|u\|_{2}$ will be large, unless $\operatorname{col}\left(u(t), t \in T_{u}\right)$ is orthogonal to the right singular vectors associated with the $n$ smallest singular values of $\Psi_{T_{u}, T_{u}}$. This control will moreover achieve that the output on the interval $T_{y}$ which equals

$$
y:=\Psi_{T_{u}, T_{y}} u=\Psi_{T_{u}, T_{y}} \Psi_{T_{u}, T_{u}}^{\dagger} y_{\mathrm{ref}}
$$

has large norm $\|y\|_{2}$, as the right singular vectors corresponding to the $n$ smallest singular values of $\Psi_{T_{u}, T_{u}}$ exactly align with the right singular vectors corresponding to the $n$ nonzero singular values of $\Psi_{T_{u}, T_{y}}$. Statement 2) of Theorem IV.1 moreover shows that this effect gets worse if the length of $T_{u}$ is increased. Hence, if one or more of the Hankel singular values of $\Psi_{T_{u}, T_{y}}$ converge to values close to one (as $N \rightarrow \infty$ ) then tracking problems with norm constraint inputs or norm constraint (future) outputs can only be solved on relatively small control intervals $T_{u}$. The maximum allowable length of these intervals is determined by the magnitude of the limiting Hankel singular values.

Another consequence of Theorem IV.1 is that any right inverse of $\Psi_{T_{u}, T_{u}}$ has an induced norm larger or equal to $\left(1-\sigma_{1}\left(\Psi_{T_{u}, T_{y}}\right)^{2}\right)^{-1 / 2}$. Moreover, a right inverse achieving this minimum norm is given by the Moore-Penrose inverse of $\Psi_{T_{u}, T_{u}}$. A further consequence of the theorem is that for $N \rightarrow \infty$ the attainable minimum norm of any inverse converges to $\left(1-\sigma_{1}\left(\Psi_{\mathbb{Z}_{-}, \mathbb{Z}_{+}}\right)^{2}\right)^{-1 / 2}$. This result suggests the following consequence of Theorem IV.1.

Proposition IV.2: The minimum $H_{\infty}$ norm of any stable right inverse of a structural inner $G \in R H_{\infty}^{p \times m}$ is equal to $\left(1-\sigma_{1}^{2}\left(\Psi_{\mathbb{Z}_{-}, \mathbb{Z}_{+}}\right)\right)^{-1 / 2}$ and there exists a stable right inverse that attains this minimum.

To show this as a direct consequence of Theorem IV.1 some technical difficulties need to be resolved which are beyond the scope of this paper. For instance one needs to show that the right inverse with minimal norm is again an operator with Toeplitz structure. Proposition IV.2 is however proven in [47] for the continuous time case based on infinite time arguments and can be proven in discrete time analogously.

An elegant expression for the singular value decomposition of $\Psi_{T_{u}, T_{u}}$ for an inner square system, is an immediate Corollary to Theorem IV.1 and given as follows.

Corollary IV.3: Let $G \in R H_{\infty}^{p \times m}$, with $p=m$ be inner with minimal input-output balanced realization $(A, B, C, D)$ and McMillan degree $n$. Let $T_{u}$ be a finite interval of length $N$, let $T_{y}:=\left\{t \in \mathbb{Z} \mid t>\max \left(T_{u}\right)\right\}$ and suppose that $N \geq n$. Then, the following hold.

1) $m N-n$ singular values of $\Psi_{T_{u}, T_{u}}$ equal one.

2) The $n$ smallest singular values of $\Psi_{T_{u}, T_{u}}$ equal the singular values of $A^{N}$.

3) The span of the right singular vectors of $\Psi_{T_{u}, T_{u}}$ corresponding to its smallest $n$ singular values equals $i \mathcal{C}_{N}^{\top}$.

4) The span of the left singular vectors of $\Psi_{T_{u}, T_{u}}$ corresponding to its smallest $n$ singular values equals $\operatorname{im} \mathcal{O}_{N}$.

Moreover, if $u$ is a control with support in $T_{u}$ and $\underline{u}:=\operatorname{col}\left(u(t) ; t \in T_{u}\right) \in \operatorname{imC}_{N}^{\top}$, then

$$
\begin{aligned}
& \left.y\right|_{T_{u}}=\Psi_{T_{u}, T_{u}} u=-\mathcal{O}_{N}\left(A^{\top}\right)^{N} P_{N}^{-1} \mathcal{C}_{N} \underline{u} \\
& \left.y\right|_{T_{y}}=\Psi_{T_{u}, T_{y}} u=\mathcal{O}\left(T_{y}\right) \mathcal{C}\left(T_{u}\right) \underline{u} .
\end{aligned}
$$

Proof: Statements 1) and 3) are proven in Theorem IV.1. The proof of statement 4) follows directly from substitution of (5) in (7). To prove (11a), substitute the identity $P_{N}=P-A^{N} P\left(A^{\top}\right)^{N}$ in (10), set $x(-N)=0$ and use that $C P A^{\top}+D B^{\top}=0$ [by (5)] to obtain that $\left.y\right|_{T_{u}}=\mathcal{O}_{N} P\left(A^{\top}\right)^{N} x$ whenever $\underline{u}=\mathcal{C}_{N}^{\top} x$. In the latter, substitute the identity $x=P_{N}^{-1} P_{N} x=P_{N}^{-1} \mathcal{C}_{N} \underline{u}$ and use that for square balanced systems $P=I$, to get (11a). By statement 2) of Theorem IV.1, the $n$ smallest singular values of $\Psi_{T_{u}, T_{u}}$ equal the square roots of the eigenvalues of $I-\Psi_{T_{u}, T_{y}}^{\top} \Psi_{T_{u}, T_{y}}$. Let $\rho_{i}$ be such a singular value $(i=1, \ldots, n)$. Then, $\rho_{i}^{2}=1-\sigma_{i}^{2}$ with $\sigma_{i}$ a corresponding singular value of $\Psi_{T_{u}, T_{y}}$. Since the system is square inner with an input-output balanced realization the gramians $P=Q=I$, and we infer from the proof of Lemma II.2 that $\sigma_{i}=\lambda_{i}^{1 / 2}\left(P_{N}\right)$. Since $P_{N}=P-A^{N} P\left(A^{\top}\right)^{N}=I-A^{N}\left(A^{\top}\right)^{N}$, it thus follows that $\rho_{i}^{2}=\lambda_{i}\left(A^{N}\left(A^{\top}\right)^{N}\right)$, i.e., for $i=1, \ldots, n, \rho_{i}$ coincides with the $i$ th singular value of $A^{N}$. This proves statement 2). Equation (11b) is a direct consequence of Lemma II.2.

For square systems, the $n$ smallest singular values of the Toeplitz operator are therefore decreasing functions of $N$ that converge to zero for $N \rightarrow \infty$. In the next section, the consequences and interpretations of the results obtained in this section will be discussed for a number of control problems.

\section{ANALYTIC TRADEOFF FOR INNER SYSTEMS}

In this section, we take a more detailed look at the consequences of the results of the previous section. For this, we consider problems 1), 2), and 3) of Definition III.1 in this section. Problem 4) will be dealt with in Section VI. 


\section{A. Tracking Control}

Consider a square inner system $G \in R H_{\infty}$, with a minimal input-output balanced realization $(A, B, C, D)$ where $D=$ $\lim _{|z| \rightarrow \infty} G(z)$ is invertible. Let $N>0$ and define the intervals $T_{u}=[-N,-1], T_{y}=[0, \infty)$ and $T_{u y}=T_{u} \cup T_{y}$. Suppose that a reference signal $y_{\text {ref }} \in \ell_{2}$ is given with support $\operatorname{supp}\left(y_{\text {ref }}\right) \subseteq$ $T_{u y}$. Consider the following tracking problem.

Definition V.I (Tracking problem): The finite time tracking problem amounts to finding a control $u \in \ell_{2}\left(T_{u}\right)$ such that $\left.y_{\mathrm{ref}}\right|_{T_{u}}=\Psi_{T_{u}, T_{u}} u$. Moreover, we wish to find a recursive expression for updating the control $u$ as a function of the length $N$ of the control interval $T_{u}$ and characterize the resulting increase in control effort.

Note that this problem is equivalent to the construction of the inverse of the Toeplitz operator $\Psi_{T_{u}, T_{u}}$. By invertibility of $\lim _{|z| \rightarrow \infty} G(z)$, this inverse exists so that a solution to the tracking problem exists and is unique. In this section, we provide a recursive solution to the tracking problem and discuss its consequences. In particular, we will be interested in the effect of the controls $u$ on the output $y(t)$ of the system for time $t \in T_{y}$ viewed as a function of the length $N$ of the control interval.

For a real matrix $A, \Pi_{A}$ will denote the orthogonal projector onto $\operatorname{im} A$ and $\Pi_{A^{\perp}}=I-\Pi_{A}$ is the projection on the orthogonal complement $[\operatorname{im} A]^{\perp}$. Let the reference signal $y_{\text {ref }}$, when restricted to $T_{u}$, be decomposed as $\left.y_{\text {ref }}\right|_{T_{u}}=y_{\text {ref1 }}+y_{\text {ref2 }}$ where

$$
\begin{aligned}
& y_{\text {ref1 }}=\left.\prod_{\mathcal{O}_{N}^{\perp}} y_{\text {ref }}\right|_{T_{u}} \\
& y_{\text {ref2 }}=\left.\prod_{\mathcal{O}_{N}} y_{\text {ref }}\right|_{T_{u}} .
\end{aligned}
$$

Then, $\left\langle y_{\text {ref1 }}, y_{\text {ref2 } 2}\right\rangle=0$ and since $\Psi_{T_{u}, T_{u}}$ is invertible, there exist unique $u_{1}$ and $u_{2}$ such that $y_{\text {ref1 }}=\Psi_{T_{u}, T_{u}} u_{1}$ and $y_{\text {ref2 }}=$ $\Psi_{T_{u}, T_{u}} u_{2}$. The following result can be established.

Theorem V.2: Under the aforementioned conditions, the following hold.

1) $\left\|u_{1}\right\|_{2}=\left\|y_{\text {ref1 } 1}\right\|_{2}$.

2) $u_{1}$ does not affect the output of the system on the time interval $T_{y}$, i.e., $\Psi_{T_{u}, T_{y}} u_{1}=0$.

3) The control $u$ solves the tracking problem if and only if $u=u_{1}+u_{2}$. Moreover, $A$ is invertible and

$$
\operatorname{col}\left(u_{2}(t), t \in T_{u}\right)=-\mathcal{C}_{N}^{\top}\left(A^{\top}\right)^{-N} Q_{N}^{-1} \mathcal{O}_{N}^{\top} y_{\text {ref2 } 2}
$$

4) Let $T_{u}^{\prime}=[-N, 0], u=u_{1}+u_{2}$ and define

$$
u^{\prime}(t)= \begin{cases}u(t) & \text { if } t \in T_{u} \\ D^{-1}\left[y_{\mathrm{ref}}(0)+C\left(A^{\top}\right)^{-N} \mathcal{O}_{N}^{\top} y_{\mathrm{ref} 2}\right], & \text { if } t=0 .\end{cases}
$$

Then, $\left.y_{\text {ref }}\right|_{T_{u}^{\prime}}=\Psi_{T_{u}^{\prime}}$, $T_{u}^{\prime} u^{\prime}$, i.e., $u^{\prime}$ solves the tracking problem on $T_{u}^{\prime}$ and is a recursive update of $u$ for the one time instant extension $T_{u}^{\prime}$ of $T_{u}$.

Proof: We first prove statement 3). Since $(A, B, C, D)$ is an input balanced realization of an inner function, Lemma II.3 implies that $A^{\top} A=I-C^{\top} C$. By invertibility of $D$, (4) yields that $C=-D^{-\top} B^{\top} A$, so that for every $x$ with $A x=0$ we must have

$$
0=\|A x\|^{2}=\|x\|^{2}-\left\|D^{-\top} B^{\top} A x\right\|^{2}=\|x\|^{2}
$$

i.e., $x=0$. Hence, $A$ must be invertible. By linearity of $\Psi_{T_{u}, T_{u}}$, $\left.y_{\text {ref }}\right|_{T_{u}}=y_{\text {ref } 1}+y_{\text {ref } 2}=\Psi_{T_{u}, T_{u}}\left(u_{1}+u_{2}\right)$, so that $u$ solves the tracking problem if and only if $u=u_{1}+u_{2}$. With Corollary IV.3 this yields statement 3 ). The properties of the singular value decomposition of $\Psi_{T_{u}, T_{u}}$, stated in Corollary IV.3, imply that $\Psi_{T_{u}, T_{u}}$ is a contraction when viewed as a map from $\operatorname{imC}_{N}^{\top}$ to $\mathrm{imO}_{N}$ and an isometry when viewed as a map from $\left[\mathrm{imC}_{N}^{\top}\right]^{\perp}$ to $\left[\mathrm{im} \mathcal{O}_{N}\right]^{\perp}$. Hence, $u_{1}=\Pi_{\mathcal{C}_{N}^{\top}} u, u_{2}=\Pi_{\mathcal{C}_{N}^{\top} \perp} u$ with $\left\langle u_{1}, u_{2}\right\rangle=$ 0 and $\left\|u_{1}\right\|_{2}=\left\|y_{\text {ref1 }}\right\|_{2}$, which proves Statement 1. Moreover, since $G$ is inner, the output $y=\Psi_{T_{u}, T_{u y}} u_{1}$ satisfies $\left\|u_{1}\right\|^{2}=$ $\|y\|^{2}=\left\|\left.y\right|_{T_{u}}\right\|^{2}+\left\|\left.y\right|_{T_{y}}\right\|^{2}$ where $\left.y\right|_{T_{u}}=y_{\text {ref1 } 1}$. It follows that $\left\|\left.y\right|_{T_{y}}\right\|$ must vanish and, hence, $\left.y\right|_{T_{y}}=\Psi_{T_{u}, T_{y}} u_{1}=0$. This proves statement 2 . To prove the fourth statement, observe that, by invertibility of $\Psi_{T_{u}, T_{u}}$ and $\Psi_{T_{u}^{\prime}, T_{u}^{\prime}}$, the solutions of the tracking problem over the interval $T_{u}$ and $T_{u}^{\prime}$ are unique. Hence, $\left.u\right|_{T_{u}}=\left.u^{\prime}\right|_{T_{u}}$. Let $y:=\Psi_{T_{u}, T_{y}} u$. Then

$$
\begin{aligned}
\operatorname{col}\left(y(t), t \in T_{y}\right) & =\mathcal{O}\left(T_{y}\right) \mathcal{C}\left(T_{u}\right) u=\mathcal{O}\left(T_{y}\right) \mathcal{C}\left(T_{u}\right) u_{2} \\
& =-\mathcal{O} P_{N}\left(A^{\top}\right)^{-N} Q_{N}^{-1} \mathcal{O}_{N}^{\top} y_{\text {ref } 2}
\end{aligned}
$$

where we used that the hypothesis on $G$ implies that $A$ is invertible. At time $t=0$, the input $u^{\prime}(0)$ should therefore satisfy

$$
y_{\mathrm{ref} 2}(0)=-C P_{N}\left(A^{\top}\right)^{-N} Q_{N}^{-1} \mathcal{O}_{N}^{\top} y_{\mathrm{ref} 2}+D u^{\prime}(0) .
$$

Solving for $u^{\prime}(0)$ and using that $P_{N}=I-A^{N}\left(A^{\top}\right)^{N}$, $Q_{N}=I-\left(A^{\top}\right)^{N} A^{N}$ yields that $u^{\prime}(0)=D^{-1} y_{\mathrm{ref}}(0)+$ $D^{-1} C\left(A^{\top}\right)^{-N} \mathcal{O}_{N}^{\top} y_{\text {ref2 }}$.

Theorem V.2 has the following interpretation. If $u$ is the unique control that solves the tracking problem, then statements 1) and 2) show that the $u_{1}$ component of $u$ will be bounded and will not affect future outputs of the system. These properties are independent of the control horizon $N$. Statement 3 ) provides an explicit expression for the $u_{2}$ component of $u$ and statement 4 ) gives a recursive update of the control for a one step extension of the control interval. Observe that the first term of $u^{\prime}(0)$ achieves instantaneous tracking of $y_{\text {ref }}(0)$, while the second term compensates the influence of past inputs on the output at time $t=0$. Whether $u^{\prime}$ is bounded, as $N \rightarrow \infty$ is determined by the second term of $u^{\prime}(0)$ and hence by the alignment of $\left.y_{\text {ref }}\right|_{T_{u}}$ to $\mathcal{O}_{N}$. Hence, $u^{\prime}$ is guaranteed to be bounded for $N \rightarrow \infty$ only if $y_{\text {ref }} \perp \mathrm{imO}$.

To investigate the case where $y_{\text {ref }} \not \perp \mathrm{imO}$, let us assume that $y_{\text {ref }} \in \operatorname{im\mathcal {O}}$. Then there exists $x_{0} \in \mathbb{R}^{n}$ such that $y_{\text {ref }}(t)=$ $C A^{t+N} x_{0}, t \in T_{u y}$. Typically, this case arises in situations where transients need to be tracked. Consider the controls $u$ and $u^{\prime}$ as defined in Theorem V.2 and let $y=\Psi_{T_{u}, T_{u y}} u$ and $y^{\prime}=\Psi_{T_{u}^{\prime}}, T_{u y} u^{\prime}$ be the corresponding outputs on $T_{u y}$. Consider the tracking errors

$$
\begin{gathered}
e=y-y_{\text {ref }} \\
e^{\prime}=y^{\prime}-y_{\text {ref }} .
\end{gathered}
$$

Since $\left.y\right|_{T_{u}}=\left.y^{\prime}\right|_{T_{u}}$ it follows that $\left.e\right|_{T_{u}}=\left.e^{\prime}\right|_{T_{u}}$. Let $T_{0}:=$ $[-N-n,-N-1]$. By controllability of the system, there exists 
a control $u_{0}: T_{0} \rightarrow \mathbb{R}^{m}$ which steers the state of (1) from state 0 at time $-N-n$ to $x_{0}$ at time $-N$. Define

$$
\begin{aligned}
& v(t):= \begin{cases}0, & \text { if } t<-N-n \\
-u_{0}(t), & \text { if } t \in T_{0} \\
u(t), & \text { if } t \in T_{u} \\
0, & \text { if } t \in T_{y}\end{cases} \\
& v^{\prime}(t):= \begin{cases}0, & \text { if } t<-N-n \\
-u_{0}(t), & \text { if } t \in T_{0}, \\
u^{\prime}(t), & \text { if } t \in T_{u}^{\prime} \\
0, & \text { if } t>0 .\end{cases}
\end{aligned}
$$

Then by construction, $e=\Psi_{\mathbb{Z}, T_{u y}} v$ and $e^{\prime}=\Psi_{\mathbb{Z}, T_{u y}} v^{\prime}$. Since the system is inner, we have that $\|e\|_{2}=\|v\|_{2}$ and $\left\|e^{\prime}\right\|_{2}=$ $\left\|v^{\prime}\right\|_{2}$. Moreover, $\left\|v^{\prime}\right\|_{2} \geq\|v\|_{2}$ and $\left.v\right|_{\mathbb{Z}_{-}}=\left.v^{\prime}\right|_{\mathbb{Z}_{-}}$from which we derive that

$$
\begin{aligned}
e^{\prime}(0) & =0 \\
\left\|e^{\prime}\right\|_{\ell_{2}\left(T_{y}\right)} & \geq\|e\|_{\ell_{2}\left(T_{y}\right)} .
\end{aligned}
$$

Hence, $u^{\prime}$ achieves instantaneous tracking of the reference signal $y_{\text {ref }}$ at time $t=0$ at the cost of a nondecreasing norm of the future tracking error. For a receding horizon real-time implementation of the controller, this means that subsequent updates of the control action may lead to an unbounded tracking error as the control horizon $N$ tends to infinity.

For an inner square system the analytic tradeoff can therefore be attributed to the subspace $i m \mathcal{O}_{N}$ in the output space and the subspace im $\mathcal{C}_{N}$ of the input space. Compensation of the transient behavior over the interval $T_{u}$ may result in a possibly unbounded tracking error.

\section{B. Deadbeat Control}

In this section, we consider a deadbeat control problem, which is, in some sense, a slight variation to the problem of Definition V.1. Let $G \in R H_{\infty}^{p \times p}$ be an inner square system, with minimal balanced realization $(A, B, C, D)$ and McMillan degree $n$. As before, let $N>0$ and define the intervals $T_{u}=[-N,-1], T_{y}=[0, \infty)$ and $T_{u y}=T_{u} \cup T_{y}$. Let $y_{\text {ref }} \in \ell_{2}$ be a reference signal, with $\operatorname{supp}\left(y_{\text {ref }}\right) \subseteq T_{u y}$. The basic idea of deadbeat is that the output of the system matches the reference signal after a predefined number of samples, say $n_{\mathrm{db}}$. Let $0<n_{\mathrm{db}}<N$ and define the time intervals $T_{u}^{-}=\left[-N,-N+n_{\mathrm{db}}-1\right]$ and $T_{u}^{+}=\left[-N+n_{\mathrm{db}},-1\right]$.

Definition V.3 (Deadbeat Problem): The deadbeat control problem amounts to finding $u \in \ell_{2}\left(T_{u}, \mathbb{R}^{p}\right)$ such that $\left.y\right|_{T_{u}^{+}}=$ $\left.y_{\mathrm{ref}}\right|_{T_{u}^{+}}$and the norm $\left\|y-y_{\mathrm{ref}}\right\|_{\ell_{2}\left(T_{y}\right)}$ is minimal. Here $y=$ $\Psi_{T_{u}, T_{u y}} u$. Moreover, we wish to find a recursive expression for updating the control $u$ as a function of the length $N$ of the control interval $T_{u}$ and characterize the resulting increase in control effort. In addition, we will be interested in finding the minimal $n_{\mathrm{db}}$ such that the deadbeat control problem is solvable for all $y_{\text {ref }} \in \ell_{2}\left(T_{u y}\right)$.

The solution to the deadbeat control problem is given in the following result.

Theorem V.4: In the above notation, let $T_{I}=$ $s^{\left(N-n_{\mathrm{db}}\right)} T_{u}^{-}=\left[-n_{\mathrm{db}},-1\right]$ and let $y_{\mathrm{ref} 1}$ and $y_{\mathrm{ref} 2}$ be as defined in (12). The deadbeat control problem is solvable if and only if $P_{n_{\mathrm{db}}}:=P\left(\left[-n_{\mathrm{db}},-1\right]\right)$ is nonsingular. In that case, a solution is given by the control $u=u_{1}+u_{2}$ where

$$
\begin{aligned}
& u_{1}(t)= \begin{cases}0, & t \in T_{u}^{-} \\
\left.\left(\Psi_{T_{u}^{+}, T_{u}^{+}}\right)^{\dagger} y_{\mathrm{ref} 1}\right|_{T_{u}^{+}}, & t \in T_{u}^{+}\end{cases} \\
& u_{2}(t)=\left\{\begin{array}{l}
\mathcal{C}\left(T_{I}\right)^{\top} P_{n_{\mathrm{db}}}^{-1} A^{n_{\mathrm{db}}} Q_{N}^{-1} \mathcal{O}_{N}^{\top} \operatorname{col}\left(y_{\mathrm{ref}}(k), k \in T_{u}\right) \\
\quad t \in T_{u}^{-} \\
0, \quad t \in T_{u}^{+} .
\end{array}\right.
\end{aligned}
$$

Proof: Define

$$
x_{0}:=Q_{N}^{-1} \mathcal{O}_{N}^{\top} \operatorname{col}\left(y_{\mathrm{ref}}(t), t \in T_{u}\right)
$$

and note that $x_{0}$ is independent of $N$ (as long as $Q_{N}$ is invertible) because there also holds that $x_{0}=Q^{-1} \mathcal{O}^{\top} \operatorname{col}\left(y_{\text {ref }}(t), t \in\right.$ $\left.T_{u y}\right)$. With the projections $\Pi_{\mathcal{O}}:=\mathcal{O} Q^{-1} \mathcal{O}^{\top}$ and $\Pi_{\mathcal{O}^{\perp}}:=$ $I-\Pi_{\mathcal{O}}$ we define

$$
y_{\text {ref1 }}:=\Pi_{\mathcal{O} \perp}\left(\left.y_{\text {ref }}\right|_{T_{u y}}\right) \quad y_{\text {ref2 }}:=\Pi_{\mathcal{O}}\left(\left.y_{\text {ref }}\right|_{T_{u y}}\right) .
$$

Then, $y_{\text {ref }}=y_{\text {ref1 } 1}+y_{\text {ref2 } 2}, y_{\text {ref2 } 2}=\mathcal{O} x_{0}$ and $\left.y_{\text {ref2 } 2}\right|_{T_{y}}=\mathcal{O} A^{N} x_{0}$. First suppose that $P_{n_{\mathrm{db}}}$ is invertible. By Lemma II.2, $\Psi_{T_{u}^{-}, T_{u}^{+}}=\mathcal{O}\left(-s T_{u}^{+}\right) \mathcal{C}\left(T_{I}\right)$, which, using (14b) and (15), yields that

$$
\Psi_{T_{u}, T_{u}^{+}} u_{2}=\left.\Psi_{T_{u}^{-}, T_{u}^{+}} u_{2}\right|_{T_{u}^{-}}=\mathcal{O}\left(-s T_{u}^{+}\right) A^{n_{\mathrm{db}}} x_{0}=\left.y_{\mathrm{ref} 2}\right|_{T_{u}^{+}} .
$$

Since $\Pi_{\mathcal{O}\left(-s T_{u}^{+}\right)^{\perp}} \Psi_{T_{u}^{+}, T_{u}^{+}}$is surjective, (14a) implies that

$$
\Psi_{T_{u}, T_{u}^{+}} u_{1}=\left.\Psi_{T_{u}^{+}, T_{u}^{+}} u_{1}\right|_{T_{u}^{+}}=\left.y_{\mathrm{ref} 1}\right|_{T_{u}^{+}} .
$$

It thus follows that $\Psi_{T_{u}, T_{u}^{+}} u=\Psi_{T_{u}, T_{u}^{+}}\left(u_{1}+u_{2}\right)=\left.y_{\mathrm{ref}}\right|_{T_{u}^{+}}$, i.e., $u$ achieves tracking of $y_{\text {ref }}$ on $T_{u}^{+}$. Moreover, by Theorem V.2, $\Psi_{T_{u}, T_{y}} u=\Psi_{T_{u}, T_{y}} u_{2}=\mathcal{O} A^{N} x_{0}=\left.y_{\text {ref } 2}\right|_{T_{y}}$, so that

$$
\begin{aligned}
\left\|\left.y_{\mathrm{ref}}\right|_{T_{y}}-\Psi_{T_{u}, T_{y}} u\right\|_{2} & =\left\|\left.y_{\mathrm{ref} 1}\right|_{T_{y}}+\left.y_{\mathrm{ref} 2}\right|_{T_{y}}-\Psi_{T_{u}, T_{y}} u_{2}\right\|_{2} \\
& =\left\|\left.y_{\mathrm{ref} 1}\right|_{T_{y}}\right\|_{2}
\end{aligned}
$$

which is minimal among all controls that achieve tracking on $T_{u}^{+}$. Second, suppose that $P\left(T_{I}\right)$ is not invertible and let $0 \neq$ $x_{1} \in \mathbb{R}^{n}$ be such that $P\left(T_{I}\right) x_{1}=0$. Define $y_{\text {ref }} \in \ell_{2}\left(T_{u}^{+} \cup T_{y}\right)$ by $\operatorname{col}\left(y_{\mathrm{ref}}(t) ; t \in T_{u}^{+} \cup T_{y}\right)=\mathcal{O} x_{1}$. Then, there does not exist a $\left.u\right|_{T_{u}^{-}}$that steers $x(-N)=0$ to $x\left(-N+n_{\mathrm{db}}\right)=x_{1}$. Hence $y_{\text {ref }}$ can not be tracked on $T_{u}^{+}$, which implies that the deadbeat control problem will not be solvable.

From Theorem V.4, we conclude that the existence of a solution to the deadbeat control problem is determined by the regularity of $P_{n_{\mathrm{db}}}$. It is well known [23] that this condition is fulfilled if $n_{\mathrm{db}} \geq n$. The minimum value of $n_{\mathrm{db}}$ for which $P_{n_{\mathrm{db}}}$ is nonsingular, say $n_{\min }$, may be smaller than $n$. Contrary to the solution of the tracking problem, the solution to the deadbeat control problem need not be unique if $n_{\mathrm{db}} \geq n_{\min }$. Also, observe that $u_{2}$ is independent of $N$. Hence, contrary to the solution of the tracking problem, we can find a control $u=u_{1}+u_{2}$ which solves the deadbeat control problem and remains bounded as $N \rightarrow \infty$. 


\section{RECEDING HORIZON CONTROL}

In MPC or, better, receding horizon control, the control problem is usually split up in two successive steps. First the steady state problem is considered and, second, a dynamic initial state problem is solved [25], [36]. The characteristic feature of receding horizon controllers is that the control strategy is determined by the optimization of a performance function on a finite time interval. This interval, the control interval, stretches from the current time to a time instant which is a fixed time-slot ahead. The optimal control is calculated and implemented only until new measurements become available. Based on the new measurements, an update of the control strategy is determined by repeating the optimization of the performance function at the next time sample. In this subsection we will be interested in the conditions for which such a receding horizon control scheme results in an exponentially stable controlled process. Stability properties of receding horizon control schemes have been the subject of many investigations. See, e.g., [10], [16], [25], [36], [44], [48], and [49], or the excellent survey paper [30]. Here, we consider a finite time linear quadratic optimization criterion with a weighted end-point penalty and analyze the stability properties of the controlled system as function of the end-point weight of the criterion function. The main result of this section is a useful and explicit parametrization of a class of end-point penalties for which exponential stability of the MPC controlled system can be guaranteed. This result appeared separately in [54].

Consider (1) and let $t \in \mathbb{Z}_{+}$be the current time, $T_{t}=[t, t+$ $N-1]$ the control interval of length (or horizon) $N>0$ and consider the quadratic objective function $J_{t}: \mathbb{R}^{n} \times\left(\mathbb{R}^{m}\right)^{T_{t}} \rightarrow$ $\mathbb{R}_{+}$defined by

$$
J_{t}\left(x_{t}, u\right):=\sum_{k \in T_{t}}\|y(k)\|^{2}+\|x(t+N)\|_{E}^{2}
$$

where $y: T_{t} \rightarrow \mathbb{R}^{p}$ is the output of the system (1) subject to the initial condition $x(t)=x_{t}$ and the input $u: T_{t} \rightarrow \mathbb{R}^{m}$. The second term in the right-hand-side of (16) is a weighted end-point penalty and is defined as $\|x\|_{E}^{2}:=\langle x, E x\rangle=x^{\top} E x$ where $E=E^{\top} \in \mathbb{R}^{n \times n}$ is a real symmetric matrix. We will not assume that $E \geq 0$ and, hence, the end-point weight may, at least in principle, be indefinite. Note that the summation in (16) ranges over a time dependent interval of fixed length $N$. Whenever finite, the optimal cost is defined as

$$
J_{t}^{\mathrm{opt}}\left(x_{t}\right):=\inf _{u: T_{t} \rightarrow \mathbb{R}^{m}} J_{t}\left(x_{t}, u\right) .
$$

The (open-loop) optimization problem at time instant $t$ amounts to minimizing (16) subject to (1). If such a minimizing control, say $u_{t}^{\text {opt }}: T_{t} \rightarrow \mathbb{R}^{m}$, exists, we have that

$$
J_{t}^{\mathrm{opt}}\left(x_{t}\right)=J_{t}\left(x_{t}, u_{t}^{\mathrm{opt}}\right) .
$$

In a receding horizon setting only the first time instant $u_{t}^{\mathrm{opt}}(t)$ of $u_{t}^{\mathrm{opt}}$ is implemented as input for (1). This means that at time $t$, $u(t)=u_{t}^{\mathrm{opt}}(t)$ is fed into (1), and the next state $x_{t+1}:=A x_{t}+$ $B u_{t}^{\text {opt }}(t)$ is taken as initial state for a renewed minimization of the criterion $J_{t+1}\left(x_{t+1}, \cdot\right)$ at the next sample time $t+1$. This calculation is repeated for every $t \in \mathbb{Z}_{+}$and results in the sequence

$$
u_{\mathrm{mpc}}:=\left\{u_{t}^{\mathrm{opt}}(t)\right\}_{t \in \mathbb{Z}_{+}}
$$

which we call the receding horizon or model predictive controller for the plant (1). This terminology and this setting is rather standard, and we emphasize that the controller (18) is not necessarily optimal in the sense that it minimizes a cost criterion. Here, we address the problem of exponential stability of the controlled system that is obtained by implementing the receding horizon controller (18) for (1).

Definition VI.1 (Stability of an MPC-scheme): The receding horizon control problem with stability is to find $E=E^{\top}$ such that the controlled system (1), (18) is exponentially stable in the sense that there exist constants $c_{1}>0$ and $0<c_{2}<1$ such that $\|x(t)\| \leq c_{1}\left\|x_{0}\right\| c_{2}^{t}$ for all $t \in \mathbb{Z}_{+}$and for all $x_{0} \in \mathbb{R}^{n}$. In that case, the control sequence $u_{\mathrm{mpc}}$ defined by (18) is called stabilizing for the plant.

For practical reasons it may be desirable to incorporate different weights of the components of the output $y$ in the criterion function (16). This means that $\|y(k)\|^{2}$ in (16) needs to be replaced by the quadratic form $\langle y(k), W y(k)\rangle$ where $W=$ $W^{\top} \geq 0$ is a nonnegative definite weighting matrix. Redefining $y$ in (1) by $y^{\prime}=W^{1 / 2} y$ implies that we may assume that this weight has been incorporated already.

Consider the recursions

$$
\begin{aligned}
\Pi_{k}= & A^{\top} \Pi_{k+1} A+C^{\top} C-\left(A^{\top} \Pi_{k+1} B+C^{\top} D\right) \\
& \times\left(B^{\top} \Pi_{k+1} B+D^{\top} D\right)^{-1}\left(B^{\top} \Pi_{k+1} A+D^{\top} C\right) \\
F_{k}= & -\left(B^{\top} \Pi_{k+1} B+D^{\top} D\right)^{-1}\left(B^{\top} \Pi_{k+1} A+D^{\top} C\right)
\end{aligned}
$$

with $\Pi_{N}=E$ and $0 \leq k<N$ and define

$$
E_{k}:=B^{\top} \Pi_{k+1} B+D^{\top} D, \quad k=0, \ldots, N-1 .
$$

If the inverses in (19) and (20) are interpreted as generalized inverses, then the matrix sequences $\left\{\Pi_{k}\right\}_{k=0}^{N},\left\{F_{k}\right\}_{k=0}^{N-1}$, and $\left\{E_{k}\right\}_{k=0}^{N-1}$ are still well defined. We have that the optimal cost satisfies $J_{t}^{\text {opt }}>-\infty$ if and only if $E_{k} \geq 0$ for $k=0, \ldots N-1$. If $E_{k} \geq 0$ for $k=0, \ldots, N-1$ and $E_{k}$ is singular for some $k$ between 0 and $N-1$, then an optimal control $u_{t}^{\text {opt }}$ that satisfies (17) is not unique. The optimal control $u_{t}^{\text {opt }}$ exists and is unique if and only if $E_{k}>0$ for $k=0, \ldots, N-1$. From now on we will assume this to be the case. ${ }^{2}$ With this assumption, a completion of the squares argument shows that

$$
J_{t}\left(x_{t}, u\right)=x_{t}^{\top} \Pi_{0} x_{t}+\sum_{k \in T_{t}}\left\|u(k)-F_{k-t} x(k)\right\|_{E_{k-t}}^{2}
$$

so that the (unique) optimal control is given by the state feedback

$$
u_{t}^{\text {opt }}(k):=F_{k-t} x(k), \quad k \in T_{t}
$$

${ }^{2}$ For the purpose of the problem treated here, this assumption is reasonable and implies that $\operatorname{col}(B, D)$ is injective (i.e., there are no redundant inputs) and $E$ satisfies $B^{\top} E B+D^{\top} D>0$. A sufficient condition for this assumption is that $E>0$ and the system $(A, B, C, D)$ is left invertible. 
with $x(k)$ the solution of (1) with $x(t)=x_{t}$. It satisfies (17) with $J_{t}^{\text {opt }}\left(x_{t}\right)=x_{t}^{\top} \Pi_{0} x_{t}$ as optimal (minimal) cost. By (18)

$$
u_{\mathrm{mpc}}(t)=F_{0} x(t), \quad t \in \mathbb{Z}_{+}
$$

is a state feedback implementation of the receding horizon control law (18). Since (1) is time invariant, $\left\{\Pi_{k}\right\}_{k=0}^{N}$ and $\left\{F_{k}\right\}_{k=0}^{N-1}$ do not depend on the current time $t$. Hence, these sequences can be calculated off-line and the MPC control law (21) is, in fact, time invariant. Exponential stability of the controlled system is, therefore, guaranteed if and only if the eigenvalues of the closed-loop state evolution matrix

$$
A_{\mathrm{cl}}:=A+B F_{0}
$$

belong to the open unit disc $|z|<1$.

It is well known [30] that the receding horizon control law will, in general, not stabilize (1) if the end-point state is not weighted in the optimization criterion, i.e., if $E=0$. The following remarks address a number of special cases in which the receding horizon controller achieves exponential stability.

Remark VI.2: The receding horizon controller (18) stabilizes (1) if the minimization in (16) is carried out subject to the endpoint constraint $x(t+N)=0$ (deadbeat control). In view of the criterion function (16), this constraint has the interpretation of an infinite weight on the final state $x(t+N)$. See, for example, the surveys of [27], [30], [25], or [37]. Obviously, this requirement is a rather strict one and is undesirable, especially when the control horizon $N$ is small. This drawback has motivated research in the direction of finite terminal weighting matrices.

Remark VI.3: In [40], it has been shown that the receding horizon control problem with stability is solved if the system (1) is stable and $E=Q$ where $Q$ is the observability gramian associated with (1). We can even obtain a stronger result. If $E=$ $\Pi \leq Q$ with $\Pi$ the (unique) nonnegative definite steady-state solution of (19) (i.e., the solution $\Pi_{k}$ of (19) for which $\Pi_{k}=$ $\Pi \geq 0$ for all $0 \leq k \leq N)$, then $F_{k}$ is independent of $k$ and $N$, and the receding horizon controller (21) will stabilize the system independent of the length $N$ of the control horizon. In particular, the receding horizon controller (18) stabilizes the system (1) independent of the length $N$ of the control horizon.

Remark VI.4: An interesting method to investigate stabilization properties of the receding horizon controller (18) is based on the monotonicity of the optimal cost $J_{t}^{\mathrm{opt}}\left(x_{t}\right)$ or, alternatively, on the monotonicity of the sequence $\left\{\Pi_{k}\right\}_{k=0}^{N}$. The papers [4], [25], [26], [28], [39] fit in this line of investigation. Properly adapted to the problem formulation here, it is shown in [4] and [28] that (18) stabilizes (1) if $E=E^{\top} \geq 0$ satisfies

$$
E>(C+D H)^{\top}(C+D H)+(A+B H)^{\top} E(A+B H)
$$

for some matrix $H$. In fact, (23) implies that $J_{t}^{\text {opt }}(x)$ serves as a Lyapunov function for the system controlled by (18). In particular, if $H$ in (23) is set to $H=F_{N-1}$, then (23) simplifies to $E>\Pi_{N-1}$ to guarantee stability of the controlled system.

There exists a popular belief that an increase of the end-point weight or an increase of the control horizon will preserve exponential stability. That is
- Claim 1: if the receding horizon controller (18) is stabilizing with $N>0$, then it is also stabilizing for all $N^{\prime} \geq N$

- Claim 2: if the receding horizon controller (18) is stabilizing with $E=E^{\top}$, then it is also stabilizing for all $E^{\prime} \geq E$.

In fact, both claims are wrong. Indeed, take

$$
\begin{aligned}
& A=\left(\begin{array}{cc}
0.7 & -0.3922 \\
0 & 0.9
\end{array}\right) \quad B=\left(\begin{array}{l}
0.6775 \\
0.3647
\end{array}\right) \\
& C=\left(\begin{array}{ll}
-0.6775 & -0.3647
\end{array}\right) \quad D=0.63 .
\end{aligned}
$$

Then $(A, B, C, D)$ is a (minimal) representation of the system

$$
G(z)=\frac{(z-10 / 7)(z-10 / 9)}{(10 z / 7-1)(10 z / 9-1)}
$$

which is inner because (4) holds with $Q=\operatorname{diag}(0.9,1.4286)$. The control law (18) is stabilizing with horizon $N \leq 5$ and end-point weight $E=Q / 4$, whereas it is not stabilizing with horizon $N^{\prime}=6>N$ and end-point weight $E=Q / 4$. This disproves claim 1. In addition, if $E=Q$ and $E^{\prime}=\operatorname{diag}(2,2)$, then $E^{\prime}>E$ and (18) turns out to be stabilizing with $N=1$ and end-point $E$, whereas it is not stabilizing with $N=1$ and end-point $E^{\prime}$. This disproves the second claim. (This also shows that the last conjecture in [4] is wrong).

In this section, we propose different, and in general weaker conditions on the terminal weighting matrix $E$ so as to guarantee stability of the controlled system. The main result of this section provides a parametrization of end-point penalties for which the receding horizon control strategy (18) is stabilizing. For this, the system is assumed to be inner.

Theorem VI.5: Suppose that (1) is an input-balanced realization of the inner $p \times m$ transfer function $G$. Let $N>0$ denote the control horizon and let $Q$ be the observability gramian defined in (4). Then, the following hold.

1) If $E-Q$ and $A$ are both nonsingular, then

$$
A_{\mathrm{cl}}=\left(X_{1}-I\right)\left(X_{1}-A A^{\top}\right)^{-1} A
$$

where $X_{1}=X_{1}^{\top}$ is a matrix which is uniquely defined by the recursion

$$
\left\{\begin{array}{l}
A X_{k} A^{\top}=X_{k+1} \\
X_{N}=(E-Q)^{-1}+I .
\end{array}\right.
$$

In particular, the receding horizon controller (18) is stabilizing if (24) has its eigenvalues in $|z|<1$. Moreover, when $Q-I \leq E<Q$ then $X_{1} \leq 0$.

2) For all $E$ satisfying

$$
E=Q+\left(A^{N-1} X\left(A^{\top}\right)^{N-1}-I\right)^{-1}
$$

where $X=X^{\top}$ commutes with $A A^{\top}$ and satisfies $X^{2}>$ $A A^{\top}$, we have that the receding horizon controller (18) achieves exponential stability of the controlled system.

Before proving these claims, we would like to emphasize the importance of the above result. First, Theorem VI.5 gives a rather straightforward recursive evaluation of the controlled system. Second, it provides a parametrization of an explicit set 
of end-point penalties $E$ for which the receding horizon controller stabilizes the system (1). Since $Q-I \leq 0$ (see the proof below for this claim), this set may include nonpositive definite end-point weights. Third, since the conditions for $X$ are independent of the control horizon $N$,

$$
\lim _{N \rightarrow \infty}\left(A^{N} X\left(A^{\top}\right)^{N}-I\right)^{-1}=-I
$$

and the right-hand side of (26) therefore converges to the negative-semidefinite matrix $Q-I$ if the control horizon $N$ tends to infinity. This means that the receding horizon control law is stabilizing with nonpositive end-point penalties, asymptotically as $N \rightarrow \infty$. Fourth, for any fixed $X$ commuting with $A A^{\top}$ and satisfying $X^{2}>A A^{\top}$, the right-hand side of (26) is a nonincreasing function of $N$. This implies that the receding horizon optimal controller stabilizes the system with decreasing end-point penalties, as the control horizon $N$ increases. Note that finding suitable $X$ commuting with $A A^{\top}$ is trivial. For instance, $X=\alpha I$ commutes with $A A^{\top}$ and satisfies $X^{2}>A A^{\top}$ for $|\alpha|>1$. It is an interesting consequence of the analysis that the recursion (25) is relevant for the derivation. As shown below, $X_{k}$ is related to $\Pi_{k}$ according to $X_{k}=\left(\Pi_{k}-Q\right)^{-1}+I$ for all $k \leq N$. The sequence $\left\{X_{k}\right\}_{k=0}^{N}$ is uniquely defined by (25) if $A$ is nonsingular.

Proof [Proof of Theorem VI.5]: Item 1: Suppose that $E-$ $Q$ and $A$ are invertible. First, eliminate $C$ and $D$ from the recursions (19) and (20) by using (4). With $\Psi_{k}:=\Pi_{k}-Q$ this yields, after some straightforward manipulation

$$
\left(\begin{array}{c}
\Psi_{k} \\
F_{k}
\end{array}\right)=\left(\begin{array}{c}
A^{\top} \\
-B^{\top}
\end{array}\right) \Psi_{k+1}\left(B B^{\top} \Psi_{k+1}+I\right)^{-1} A
$$

Hence

$$
\begin{aligned}
A+B F_{k} & =A-B B^{\top} \Psi_{k+1}\left(B B^{\top} \Psi_{k+1}+I\right)^{-1} A \\
& =\left[I-B B^{\top} \Psi_{k+1}\left(B B^{\top} \Psi_{k+1}+I\right)^{-1}\right] A \\
& =\left(B B^{\top} \Psi_{k+1}+I\right)^{-1} A .
\end{aligned}
$$

Since (1) is an input-balanced isometric state representation, there holds $B B^{\top}=I-A A^{\top}$ which we use to eliminate $B B^{\top}$ from the $\Psi_{k}$ recursion. We now claim that all elements of the sequence $\left\{\Psi_{k}\right\}_{k=0}^{N}$ are invertible. Indeed, $\Psi_{N}=\Pi_{N}-Q=$ $E-Q$ is invertible, and with the induction hypothesis that $\Psi_{k+1}$ is invertible for $0<k<N$, we have that

$$
\begin{aligned}
\Psi_{k} & =A^{\top} \Psi_{k+1}\left(\left[I-A A^{\top}\right] \Psi_{k+1}+I\right)^{-1} A \\
& =A^{\top}\left(I-A A^{\top}+\Psi_{k+1}^{-1}\right)^{-1} A \\
& =\left(A^{-1}\left[\Psi_{k+1}^{-1}+I\right]\left(A^{\top}\right)^{-1}-I\right)^{-1}
\end{aligned}
$$

i.e., $\Psi_{k}$ is invertible. Hence, $\Psi_{k}$ is invertible for all $0 \leq k \leq N$. In addition, the latter expression yields that

$$
A\left(\Psi_{k}^{-1}+I\right) A^{\top}=\Psi_{k+1}^{-1}+I
$$

from which it follows that the sequence $X_{k}:=\Psi_{k}^{-1}+I, X_{N}=$ $(E-Q)^{-1}+I, k=0, \ldots, N$ is well defined and uniquely determined by the recursion (25). In addition

$$
\begin{aligned}
A+B F_{k} & =\left(B B^{\top} \Psi_{k+1}+I\right)^{-1} A \\
& =\left(\left(I-A A^{\top}\right) \Psi_{k+1}+I\right)^{-1} A \\
& =\left(\left(\Psi_{k+1}+I\right)-A A^{\top} \Psi_{k+1}\right)^{-1} A \\
& \left.=\left(\left(\Psi_{k+1}^{-1}+I\right)-I\right)\left(\Psi_{k+1}^{-1}+I\right)-A A^{\top}\right)^{-1} A \\
& =\left(X_{k+1}-I\right)\left(X_{k+1}-A A^{\top}\right)^{-1} A
\end{aligned}
$$

which, using (22), yields (24) by setting $k=0$.

We further claim that the sequence $\left\{X_{k}\right\}_{k=0}^{N}$ consists of nonpositive elements whenever $Q-I \leq E<Q$. To see this, first observe that for input-balanced isometric state representations $Q \leq I$. Indeed, since $P=I$, Lemma II.3 and Lemma II.2 (applied for $T_{u}=\mathbb{Z}_{-}$and $T_{y}=\mathbb{Z}_{+}$) imply that $\lambda_{\max }^{1 / 2}(Q)=$ $\lambda_{\max }^{1 / 2}(P Q) \leq 1$ which yields that $Q-I \leq 0$. Consequently

$$
\begin{aligned}
Q-I \leq E<Q & \Longleftrightarrow 0<Q-E \leq I \Longleftrightarrow(Q-E)^{-1} \geq I \\
& \Longleftrightarrow X_{N}=(E-Q)^{-1}+I \leq 0 .
\end{aligned}
$$

However, if $X_{N} \leq 0$, then (25) guarantees that $X_{k} \leq 0$ for all $0 \leq k \leq N$.

Item 2: Suppose that $X=X^{\top}$ commutes with $A A^{\top}$ and that $X^{2}>A A^{\top}$. First, consider the case where $A$ is invertible. Define, for $k=1, \ldots, N$, the matrices $X_{k}:=A^{k-1} X\left(A^{\top}\right)^{k-1}$ (with $X_{1}=X$ ) and note that (26) implies that $E-Q$ is invertible. Hence, the sequence $X_{k}$ is the unique solution of the recursion (25) and we infer from item 1 that

$$
A_{\mathrm{cl}}=(X-I)\left(X-A A^{\top}\right)^{-1} A .
$$

Define $Y=A A^{\top}$. From (27), it is clear that $A_{\mathrm{cl}}$ is asymptotically stable whenever

$$
A_{\mathrm{cl}} A_{\mathrm{cl}}^{\top}=(X-I)(X-Y)^{-1} Y(X-Y)^{-1}(X-I)<I .
$$

(This condition is equivalent to saying that $V(x)=x^{\top} x$ is a Lyapunov function of the controlled system). The assumption that $X$ commutes with $Y$ implies that all matrices in the latter expression commute. The proof is therefore complete if we establish the (equivalent) inequality

$$
(X-Y)^{-1}(X-I)^{2} Y(X-Y)^{-1}<I .
$$

Now, observe that

$$
\begin{aligned}
X^{2}>Y \Leftrightarrow & (I-Y)^{1 / 2} X^{2}(I-Y)^{1 / 2} \\
& >(I-Y)^{1 / 2} Y(I-Y)^{1 / 2} \\
\Leftrightarrow & X^{2}(I-Y)>Y(I-Y) \\
\Leftrightarrow & X^{2}(Y-I)<Y(Y-I) \\
\Leftrightarrow & X^{2} Y+Y<X^{2}+Y^{2} \\
\Leftrightarrow & (X-I)^{2} Y<(X-Y)^{2}
\end{aligned}
$$

which yields (28) after a straightforward substitution. It follows that $A_{\mathrm{cl}}$ has its eigenvalues in $|z|<1$ and, hence, the receding horizon controller (18) is stabilizing. 
If $A$ is not invertible, we apply a perturbation argument to establish that the expression (27) also holds in this case so that the same proof applies. Specifically, we claim that, for $\varepsilon>0$, there exists a matrix

$$
M_{\varepsilon}:=\left(\begin{array}{cc}
A_{\varepsilon} & B_{\varepsilon} \\
C_{\varepsilon} & D_{\varepsilon}
\end{array}\right)
$$

such that: i) $A_{\varepsilon}$ is invertible, ii) $M_{\varepsilon}$ defines an input-balanced realization of an inner transfer function $G_{\varepsilon} \in R H_{\infty}$, and iii) $\lim _{\varepsilon \rightarrow 0} M_{\varepsilon}=\left(\begin{array}{ll}A & B \\ C & D\end{array}\right)$.

To prove the existence of such $M_{\varepsilon}$, note that for an outputbalanced inner system $(A, B, C, D)$ we have, by Lemma II.3

$$
\left(\begin{array}{ll}
A & B \\
C & D
\end{array}\right)^{\top}\left(\begin{array}{ll}
A & B \\
C & D
\end{array}\right)=\left(\begin{array}{ll}
I & 0 \\
0 & I
\end{array}\right) .
$$

Let $F_{\varepsilon}$ be a family of state feedbacks parameterized by $\varepsilon$ with the property that $A+B F_{\varepsilon}$ is invertible for all $\varepsilon>0$ and $F_{\varepsilon} \rightarrow 0$ as $\varepsilon \downarrow 0$. Define a new system

$$
\begin{aligned}
\left(\begin{array}{ll}
A_{\varepsilon} & B_{\varepsilon} \\
C_{\varepsilon} & D_{\varepsilon}
\end{array}\right):= & \left(\begin{array}{ll}
A & B \\
C & D
\end{array}\right)\left(\begin{array}{cc}
I & -F_{\varepsilon}^{\top} \\
F_{\varepsilon} & I
\end{array}\right) \\
& \times\left(\begin{array}{cc}
\left(I+F_{\varepsilon}^{\top} F_{\varepsilon}\right)^{-1 / 2} & 0 \\
0 & \left(I+F_{\varepsilon} F_{\varepsilon}^{\top}\right)^{-1 / 2}
\end{array}\right) .
\end{aligned}
$$

Then

$$
\left(\begin{array}{ll}
A_{\varepsilon} & B_{\varepsilon} \\
C_{\varepsilon} & D_{\varepsilon}
\end{array}\right)^{\top}\left(\begin{array}{ll}
A_{\varepsilon} & B_{\varepsilon} \\
C_{\varepsilon} & D_{\varepsilon}
\end{array}\right)=\left(\begin{array}{cc}
I & 0 \\
0 & I
\end{array}\right)
$$

and $A_{\varepsilon}=\left(A+B F_{\varepsilon}\right)\left(I+F_{\varepsilon}^{\top} F_{\varepsilon}\right)^{-1 / 2}$ is clearly invertible. Moreover, $\lim _{\varepsilon \downarrow 0}\left(\begin{array}{cc}A_{\varepsilon} & B_{\varepsilon} \\ C_{\varepsilon} & D_{\varepsilon}\end{array}\right)=\left(\begin{array}{cc}A & B \\ C & D\end{array}\right)$. This shows that an output-balanced inner system can be perturbed to yield an output-balanced inner system which has no poles in the origin.

To obtain the same result for input-balanced inner systems we first transform the system into an output-balanced inner system by the state transformation $x \mapsto Q^{-1 / 2} x$. Next apply the above transformation and then transform the system back with the state transformation $x \mapsto P_{\varepsilon}^{1 / 2} x$ where $P_{\varepsilon}$ is the unique solution of $A_{\varepsilon} P_{\varepsilon} A_{\varepsilon}^{\top}+B_{\varepsilon} B_{\varepsilon}^{\top}=P_{\varepsilon}$. Here, $P_{\varepsilon}$ depends continuously on $\varepsilon$ since we can guarantee that the eigenvalues of $A_{\varepsilon}$ are inside the unit circle and stay away from the boundary. Hence, the solutions $P_{\varepsilon}$ stay away from singularities and will depend continuously on $\varepsilon$. But then it is clear that transformation into the output-balanced form is approximately the inverse of the transformation back into the input-balanced form and we obtain an arbitrary small perturbation of an input-balanced system which has no poles in the origin. This proves the claim.

Now, let $E$ be given by (26) where $X$ satisfies the hypothesis. Let $Q_{\varepsilon}$ satisfy (4) for the perturbed system defined by $M_{\varepsilon}$ and let $E_{\varepsilon}$ be the perturbed end-point weight defined by (26) with $A$ and $Q$ replaced by $A_{\varepsilon}$ and $Q_{\varepsilon}$, respectively. Let $\Pi_{\varepsilon, k}, F_{\varepsilon, k}$ be the sequences (19) and (20) for the perturbed system parameters $M_{\varepsilon}$ and where we set $\Pi_{\varepsilon, N}=E_{\varepsilon}$. For $\varepsilon$ sufficiently small, $\Pi_{\varepsilon, k}$ and $F_{\varepsilon, k}$ are well defined and $Q_{\varepsilon}$ is the unique positive-definite solution of the Lyapunov equation

$$
A_{\varepsilon}^{\top} Q_{\varepsilon} A_{\varepsilon}+C_{\varepsilon}^{\top} C_{\varepsilon}=Q_{\varepsilon} .
$$

For $\left(A_{\varepsilon}, C_{\varepsilon}\right) \rightarrow(A, C)$ this Lyapunov equation retains unique solvability from which we infer that $\lim _{\varepsilon \rightarrow 0} Q_{\varepsilon}=Q$. (Indeed, the Lyapunov equation in $Q_{\varepsilon}$ can be rewritten as $L_{\varepsilon} q_{\varepsilon}=s_{\varepsilon}$ where the vector $q_{\varepsilon}$ consists of the elements in $Q_{\varepsilon}$, and where both $L_{\varepsilon}$ and its $\operatorname{limit}_{\lim _{\varepsilon \rightarrow 0}} L_{\varepsilon}$ are injective. The limit $\lim _{\varepsilon \rightarrow 0} q_{\varepsilon}$ therefore exists and we infer that $\lim _{\varepsilon \rightarrow 0} q_{\varepsilon}=q$ with $q$ the vector of elements in $Q$ ). We have that

$$
x_{k}^{\top} \Pi_{\varepsilon, k} x_{k}=\inf _{u} \sum_{t=k}^{N-1}\left\|y_{\varepsilon}(t)\right\|_{2}^{2}+\left\|x_{\varepsilon}(N)\right\|_{E}^{2}
$$

where $\left(y_{\varepsilon}, x_{\varepsilon}\right)$ denote the output and the state of the perturbed system subject to initial condition $x_{k}$ at time $t=k$. It follows that $M_{\varepsilon} \rightarrow M$ implies that for every bounded input $u$, $x_{\varepsilon}(t) \rightarrow x(t)$ and $y_{\varepsilon}(t) \rightarrow y(t)$ pointwise in $t$ as $\varepsilon \rightarrow 0$. Consequently, $\lim _{\varepsilon \rightarrow 0} \Pi_{\varepsilon, k}=\Pi_{k}$ and in particular $\lim _{\varepsilon \rightarrow 0} F_{\varepsilon, 0}=$ $F_{0}$. Now, define $A_{\varepsilon, \mathrm{cl}}=A_{\varepsilon}+B_{\varepsilon} F_{\varepsilon, 0}$. Since $E-Q$ is nonsingular (by hypothesis), also $E_{\varepsilon}-Q_{\varepsilon}$ is nonsingular for $\varepsilon$ sufficiently small. Hence, by item 1 ), for sufficiently small $\varepsilon$ there exist real symmetric matrices $X_{\varepsilon, k}, k=0, \ldots, N$, which satisfy $A_{\varepsilon} X_{\varepsilon, k} A_{\varepsilon}^{\top}=X_{\varepsilon, k+1}$ with $X_{\varepsilon, N}=\left(E_{\varepsilon}-Q_{\varepsilon}\right)^{-1}+I$. Using the same arguments as in item 1), it follows that $X_{\varepsilon, k}=$ $\left(\Pi_{\varepsilon, k}-Q_{\varepsilon}\right)^{-1}+I$ and we infer from the definition of $E_{\varepsilon}$ that $X_{\varepsilon, 1}=X$. In particular, it follows that $\lim _{\varepsilon \rightarrow 0} X_{\varepsilon, 1}=$ $\left(\Pi_{1}-Q\right)^{-1}+I=X$, where $\Pi_{1}=\lim _{\varepsilon \rightarrow 0} \Pi_{\varepsilon, 1}$. Also, by item 1)

$$
A_{\varepsilon, \mathrm{cl}}=\left(X_{\varepsilon, 1}-I\right)\left(X_{\varepsilon, 1}-A_{\varepsilon} A_{\varepsilon}^{\top}\right)^{-1} A_{\varepsilon} .
$$

Now, conclude that

$$
\begin{aligned}
A_{\mathrm{cl}} & =\lim _{\varepsilon \rightarrow 0} A_{\varepsilon}+B_{\varepsilon} F_{\varepsilon, 0}=\lim _{\varepsilon \rightarrow 0} A_{\varepsilon, \mathrm{cl}} \\
& =\lim _{\varepsilon \rightarrow 0}\left(X_{\varepsilon, 1}-I\right)\left(X_{\varepsilon, 1}-A_{\varepsilon} A_{\varepsilon}^{\top}\right)^{-1} A_{\varepsilon} \\
& =\lim _{\varepsilon \rightarrow 0}(X-I)\left(X-A_{\varepsilon} A_{\varepsilon}^{\top}\right)^{-1} A_{\varepsilon} \\
& =(X-I)\left(X-A A^{\top}\right)^{-1} A
\end{aligned}
$$

i.e., $A_{\mathrm{cl}}$ satisfies (27).

Remark VI.6: The assumption, stated in Theorem VI.5, that the isometric state representation (1) need to be input-balanced has been made to simplify equations but does not incur any loss of generality. In the proof of Theorem VI.5 we only used that the controllability gramian $P=I$ (we did not use that $Q$ is diagonal). If (1) is not input-balanced then a convenient choice of basis of the state space transforms (1) into an input-balanced representation. Alternatively, for isometric state representations for which $P \neq I$, (25) and (24), take the form

$$
\begin{aligned}
A X_{k} A^{\top} & =X_{k+1}, \quad X_{N}=(E-Q)^{-1}+P \\
A_{\mathrm{cl}} & =\left(X_{1}-P\right)\left(X_{1}-A P A^{\top}\right)^{-1} A \\
E & =Q+\left(A^{N-1} X\left(A^{\top}\right)^{N-1}-P\right)^{-1}
\end{aligned}
$$

and $X_{k}$ is related to $\Pi_{k}$ in (19) according to $X_{k}=\left(\Pi_{k}-Q\right)^{-1}+$ $P$. 

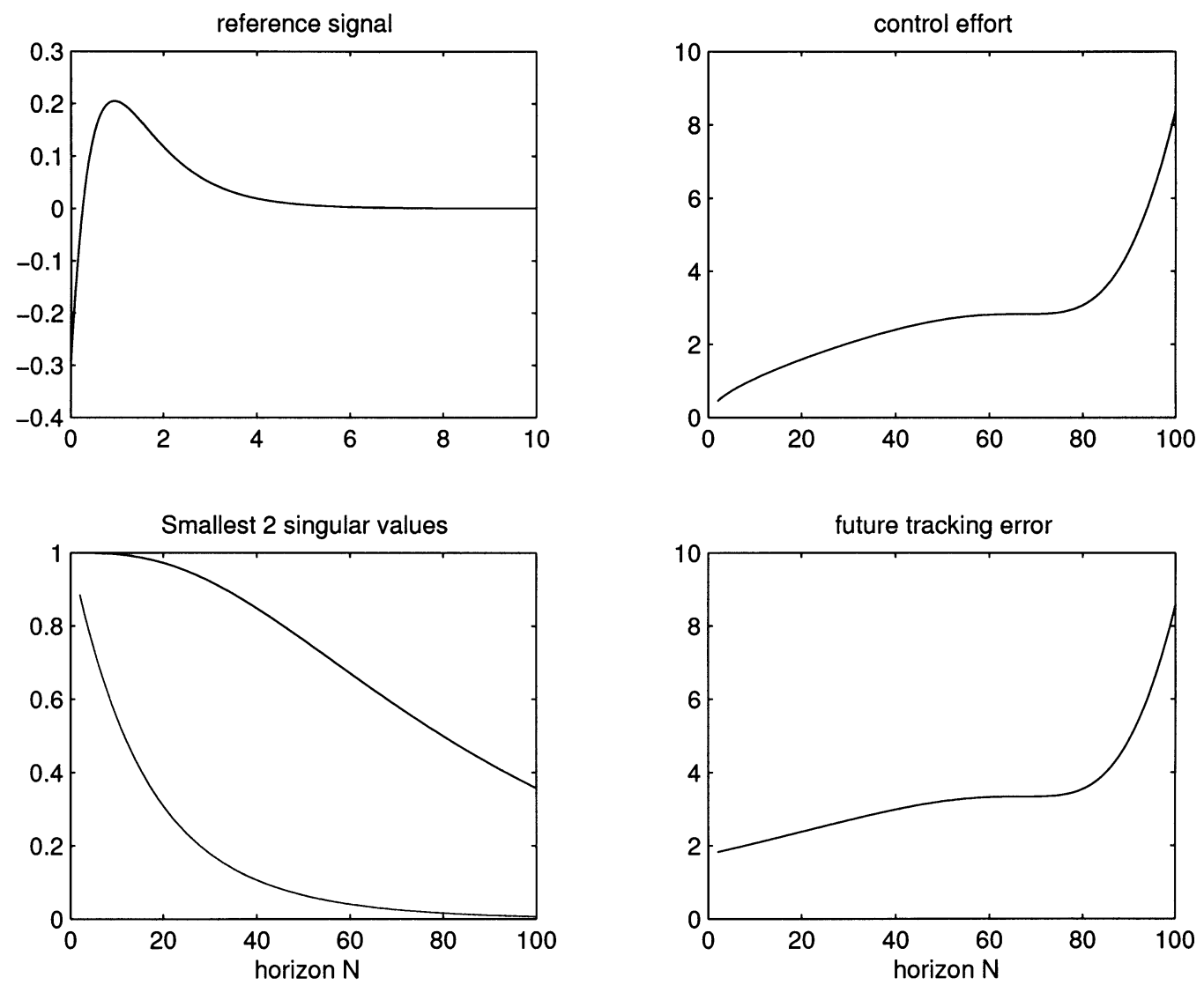

Fig. 1. Reference signal $\bar{y}_{\text {ref }}$ as function of time; control effort $\|u\|_{\ell_{2}\left(T_{u}\right)}$, singular values of $\Psi_{T_{u}, T_{u}}$ and tracking error $\|e\|_{\ell_{2}(N, \infty)}$ as functions of the control horizon $N$

\section{SiMULATION}

As an illustration of the theory of the preceding sections, consider the stable and nonminimum phase plant

$$
P(s)=\frac{(s-1)(s-2)}{s^{2}+s+2} .
$$

The continuous time model is discretized using a bilinear Tustin transformation with sample time $T_{\mathrm{s}}=0.02$ time-units, to yield a second order transfer function $P(z)$ which maps the input $\bar{u}$ to the output $\bar{y}$. As described in Section III, the graph of $P$ can be represented by

$$
\operatorname{graph}(P)=\operatorname{im}\left(\begin{array}{c}
P \\
I
\end{array}\right)=\operatorname{im} \underbrace{\left(\begin{array}{c}
G_{\bar{y}} \\
G_{\bar{u}}
\end{array}\right)}_{G}=\operatorname{im} G
$$

where $G \in R H_{\infty}^{2 \times 1}$ is an inner transfer function mapping $u$ to $y=\operatorname{col}(\bar{y}, \bar{u})$. Here, the variable $u$ is related to the input $\bar{u}$ of the plant according to $\bar{u}=G_{\bar{u}} u$ where, since $P$ is stable, $G_{\bar{u}}$ will be stable with a stable inverse. Hence, $G_{\bar{u}}$ defines a bijection between $u$ and $\bar{u}$, whereas $G_{\bar{y}} G_{\bar{u}}^{-1}$ is a normalized coprime factorization of $P(z)$ (cf. [53]). An input balanced isometric state-space representation of $G$ is given by (1) with

$$
\begin{array}{ll}
A=\left(\begin{array}{ll}
0.9992 & -0.0198 \\
0.0294 & 0.9558
\end{array}\right) & B=\left(\begin{array}{r}
0.0358 \\
-0.2926
\end{array}\right) \\
C=\left(\begin{array}{ll}
-0.0078 & 0.2422 \\
-0.0272 & 0.0560
\end{array}\right) & D=\left(\begin{array}{l}
0.6707 \\
0.6983
\end{array}\right)
\end{array}
$$

and gramians $P=I$ and $Q=\operatorname{diag}(0.8495,0.7185)$. We wish the output $\bar{y}$ of the plant to track a given reference signal $\bar{y}_{\text {ref }}$ on a time interval $T_{u}=[1, N]$ by designing a control law of the form $u(t)=F_{0} x(t)+v(t)$. Here, $F_{0}$ is a receding horizon feedback law that should not destabilize the system, while $v$ is a new auxiliary input. We take $N=25$ samples (control horizon of 0.5 time-unit) and design $F_{0}$ by means of the recursions (19) and (20) of Section VI. With $E=0$ the feedback (21) will destabilize this system, but with $E=Q$ it stabilizes (cf. Remark VI.3). To find less restrictive end-point weights which result in stabilizing feedbacks, Theorem VI.5 is applied with $X=-4 I$. $E$ defined by (26) then satisfies $0<E<Q$ and the recursions (19) and (20) yield the feedback $F_{0}=10^{-2}(0.5534-5.7059)$ which is guaranteed to stabilize $G$ and therefore also $P$.

With $F_{0}$ specified, let $G^{\prime} \in R H_{\infty}$ be the (stable) transfer function that maps $v$ to $\bar{y}$ and let $G^{\prime}=G_{\mathrm{i}} G_{\mathrm{O}}$ be an inner-outer factorization of $G^{\prime}$ where the (second order) inner factor is represented by an input-output balanced realization $\left(A_{\mathrm{i}}, B_{\mathrm{i}}, C_{\mathrm{i}}, D_{\mathrm{i}}\right)$ (cf. [13]). Consider the tracking problem on $T_{u}$ with reference signal $\bar{y}_{\text {ref }}(t)=C_{\mathrm{i}} A_{\mathrm{i}}^{(t-1)} x_{0}$ for some random $x_{0}$. Note that this signal is notoriously difficult to track as $N \rightarrow \infty$ according to the results of Theorem V.2. The two smallest singular values of the Toeplitz operator $\Psi_{T_{u}, T_{u}}$ associated with $G_{\mathrm{i}}$, the control effort $\|u\|_{2}$ to track $\bar{y}_{\text {ref }}$ on $T_{u}$ and the norm $\|e\|_{2}$ of the tracking error on $(N, \infty)$ are depicted, as functions of $N$, in Fig. 1 for $N=2, \ldots, 100$. By Theorem IV.1 the singular values are decreasing functions of 

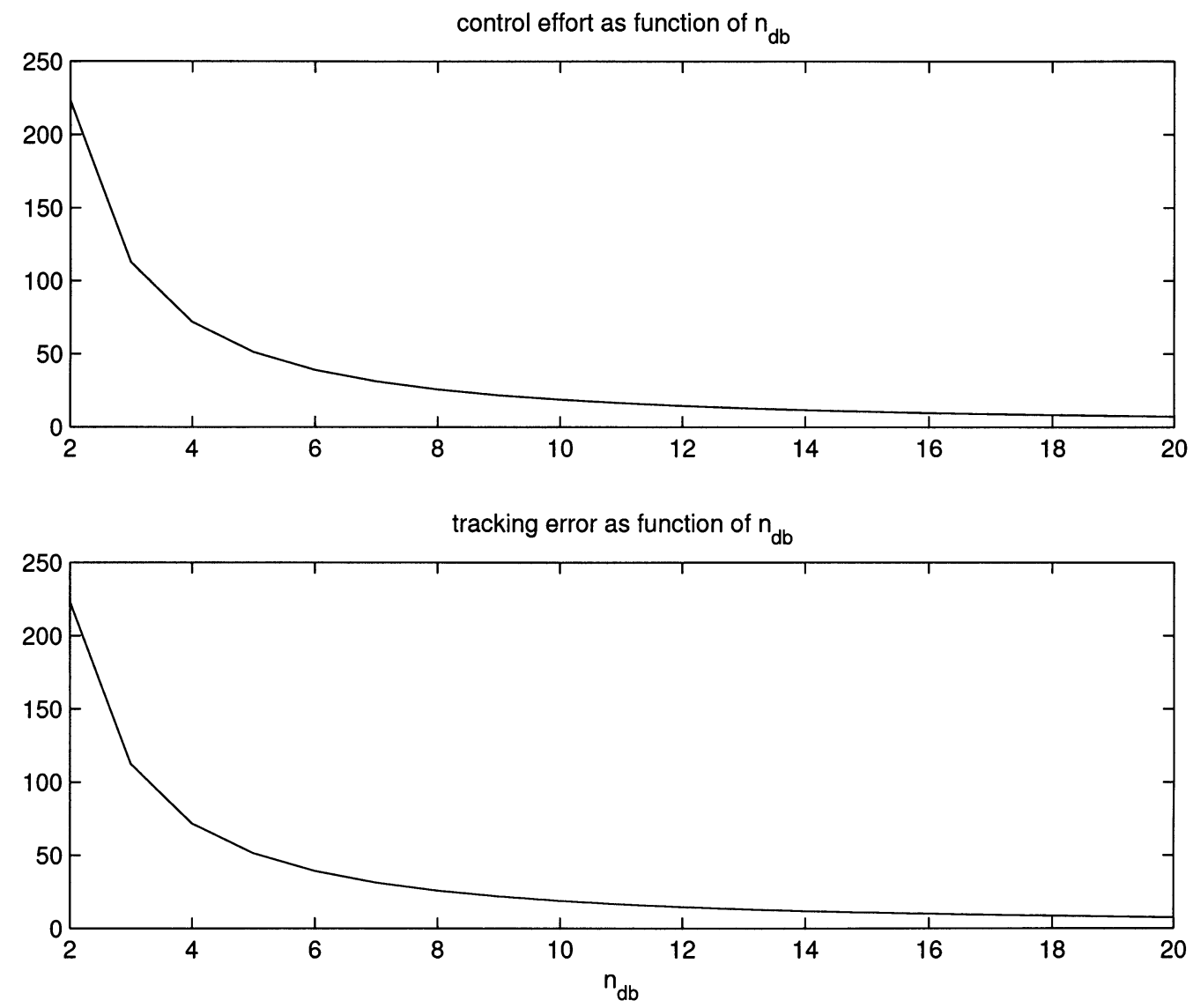

Fig. 2. Control effort $\|u\|_{2}$ and tracking error $\|e\|_{2}$ as function of the deadbeat horizon $n_{\mathrm{db}}$.

$N$, and the simulation clearly shows that the control effort and the tracking error increase without bound as functions of the control horizon $N$.

Next, consider the deadbeat problem with the same reference signal and fixed control horizon $N=100$ (two time-units). Since $P_{n_{\mathrm{db}}}$ is nonsingular if and only if $n_{\mathrm{db}} \geq 2$, the deadbeat problem will be solvable if and only if $n_{\mathrm{db}} \geq 2$. In Fig. 2, the influence of the choice of the deadbeat horizon $n_{\mathrm{db}}$ on the tracking error $\|e\|_{2}$ and the control effort $\|u\|_{\ell_{2}\left(T_{u}\right)}$ is depicted. These norms are decreasing functions of $n_{\mathrm{db}}$, i.e., the longer the deadbeat horizon, the less control effort is needed and the smaller the tracking error will be. This is in accordance with the conclusions of Theorem V.4.

\section{CONCLUSION}

In this paper, the finite time behavior of square inner systems with nonminimum phase zeros has been studied. The motivation for this work lies in the question to assess the effect of the open-loop zero structure of a dynamical system on the invertibility, tracking and stabilizability properties of a controlled system. This question is particularly relevant for applications where model predictive control techniques are used. It has been shown that the solvability of a number of relevant tracking problems depend on invertibility of a Toeplitz-like operator, defined on a signal space of input signals with finite support. The invertibility of this operator has been characterized in terms of its singular values and singular vectors. In particular, it has been shown that any such Toeplitz operator defines a finite dimensional space of reference signals which are difficult to track in the sense that: 1) the norm of the control input signal which achieves exact tracking of the reference signal increases without bound as the tracking interval increases, and 2) the norm of the tracking error outside the tracking interval increases without bound as the tracking interval increases.

For tracking problems with norm-constrained signals, this result has the interesting consequence that it characterizes a class of nonfeasible reference signals and nonfeasible tracking intervals. Typically, these nonfeasible reference signals include transients of a system.

To remedy this problem, we considered a deadbeat control problem which amounts to tracking a given reference signal after a delay of a finite number of time samples. The precise delays for which the norms of control inputs and tracking errors remain bounded have been characterized.

As a third application, we considered the exponential stability of an inner system when controlled by a receding horizon controller. More specifically, we considered a finite horizon quadratic criterion function with a variable end-point state weighting. A receding horizon implementation of the control law which minimizes this criterion may or may not be stabilizing, depending on the end-point weight. A parametrization of a set of end-point weighting matrices has been given that guarantee exponential stability of the controlled system. This parametrization includes a rich class of end-point weights and is believed to be less conservative than a number of results that 
appeared in the literature. In particular, it has been shown that the receding horizon control law is asymptotically stabilizing with nonpositive end-point weights as the length of the control horizon tends to infinity.

\section{ACKNOWLEDGMENT}

The authors would like to thank J. Hauser (University of Colorado at Boulder) for expressing his confidence that claim 2 in Section VI is wrong.

\section{REFERENCES}

[1] D. D. O. Anderson and S. Vongpanitlerd, Network Analysis and Synthesis: A Modern Systems Theory Approach. Upper Saddle River: Prentice-Hall, 1973.

[2] Y. Arkun, "Dynamic process operability. Import problems, recent results and new challenges," in CACHE, Chem. Proc. Cont. (CPC III), M. Morari and T. J. McAvoy, Eds: Elsevier, 1986, pp. 323-367.

[3] T. Backx, O. Bosgra, and W. Marquardt, "Toward intentional dynamics in supply chain conscious process operations," presented at the The FOCAP'98, Snowbird, UT, 1998, Preprint.

[4] R. R. Bitmead, M. R. Gevers, and I. R. Petersen, "Monotonicity and stabilizabiliy properties of solutions of the Riccati difference equation: Propositions, lemmas, theorems, fallacious conjectures and counterexamples," Syst. Control Lett., vol. 5, pp. 309-315, 1985.

[5] H. W. Bode, Network Analysis and Feedback Amplifier Design. New York: Van Nostrand, 1945.

[6] S. P. Boyd and C. A. Desoer, "Subharmonic functions and performance bounds on linear time-invariant feedback systems," IMA J. Math. Cont. Info., vol. 2, pp. 153-170, 1985.

[7] R. W. Brockett and M. D. Mesarovic, "The reproducibility of multivariable systems," J. Math. Anal. Applicat., vol. 11, pp. 548-563, 1965.

[8] D. Cox, J. Little, and D. O'Shara, "Ideals, varieties and algorithms: An introduction to computational algebraic geometry and commutative algebra," in Undergraduate Texts in Mathematics. New York: SpringerVerlag, 1992.

[9] J. Chen, "Multivariable gain phase and sensitivity integral relations and design tradeoffs," IEEE Trans. Automat. Contr., vol. 43, pp. 373-385, Mar. 1998.

[10] H. Chen and F. Allgöwer, "A quasiinfinite horizon nonlinear model predictive control scheme with guaranteed stability," Automatica, vol. 34, no. 10 , pp. $1205-1217,1998$.

[11] S. Fararooy, J. D. Perkins, T. I. Malik, M. J. Oglesby, and S. Williams, "Process controllability toolbox (PCTB)," Comput. Chem. Eng., vol. 17, no. 5/6, pp. 617-625, 1993

[12] B. A. Francis and G. Zames, "On $H_{\infty}$ optimal sensitivity theory for SISO feedback systems," IEEE Trans. Automat. Contr., vol. AC-29, pp. 9-19, Jan. 1984.

[13] B. A. Francis, "A course in $H_{\infty}$ control theory," in Lecture Notes in Control and Information Sciences. New York: Springer-Verlag, 1987, vol. 88 .

[14] J. S. Freudenberg and D. P. Looze, "Right half plane poles and zeros and design tradeoffs in feedback systems," IEEE Trans. Automat. Contr., vol. AC-30, pp. 555-565, June 1985.

[15] — - "Frequency domain properties of scalar and multivariable feedback systems," in Lecture Notes in Control and Information Sciences. Berlin, Germany: Springer-Verlag, 1988, vol. 104.

[16] C. E. García and M. Morari, "Internal model control 1. A unifying review and some new results," Ind. Eng. Chem. Proc. Des. Dev., vol. 21, pp. 308-323, 1982.

[17] T. T. Georgiou and M. C. Smith, "Graphs, causality, and stabilizability: linear, shift-invariant systems on $L_{2}[0, \infty)$," Math. Control Signals Syst., vol. 6, pp. 195-223, 1994.

[18] P. S. C. Heuberger, "On approximate system identification with system based orthonormal functions," Ph.D. dissertation, Delft Univ. Technol., Delft, The Netherlands, 1991.

[19] B. R. Holt and M. Morari, "Design of resilient processing plants V. The effect of deadtime on the dynamic resilience," Chem. Eng. Sci., vol. 40, no. 7, pp. 1229-1237, 1985.

[20] _ - "Design of resilient processing plants VI. The effect of right half plane zeros on the dynamic resilience," Chem. Eng. Sci., vol. 40, no. 1, pp. 59-74, 1985 .
[21] I. M. Horowitz, Synthesis of Feedback Systems. New York: Academic, 1963

[22] J. Ingham, I. J. Dunn, E. Heinzle, and J. E. Přenosil, Chemical Engineering Dynamics: An Introduction to Modeling and Computer Simulation. Weinheim, Germany: WILEY-VCH, 2000.

[23] T. Kailath, Linear Systems. Upper Saddle River, NJ: Prentice-Hall, 1980

[24] J. L. A. Koolen, "Plant operation in the future," Comput. Chem. Eng., vol. 18, pp. S477-S481, 1994.

[25] W. H. Kwon and A. E. Pearson, "A modified quadratic cost problem and feedback stabilization of a linear system," IEEE Trans. Automat. Contr., vol. AC-22, pp. 838-842, Oct. 1977.

[26] W. H. Kwon and K. B. Kim, "On stabilization receding horizon controls for linear continuous time-invariant systems," IEEE Trans. Automat. Contr., vol. 45, pp. 1329-1334, 2000.

[27] J. H. Lee, "Recent advances in model predictive control and other related areas," in The Proceedings Chemical Process Control-V, Tahoe City, UT, Jan. 7-12, 1996, Preprints

[28] J. W. Lee, W. H. Kwon, and J. Choi, "On stability of constrained receding horizon control with finite termal weighting matrix," Automatica, vol. 34, pp. 1607-1612, 1998.

[29] J. H. A. Ludlage, "Controllability analysis of industrial processes: Toward the industrial application," Ph.D. dissertation, Eindhoven Univ. Technol., Eindhoven, The Netherlands, 1997.

[30] D. Q. Mayne, J. B. Rawlings, C. V. Rao, and P. O. M. Scokaert, "Constrained model predictive control: Stability and optimality," Automatica, vol. 36, pp. 789-814, 2000.

[31] T. E. Marlin and T. J. McAvoy, "A short-cut method for process control and operability analysis," in CACHE, Chem. Proc. Cont. (CPC III), M. Morari and T. J. McAvoy, Eds. New York: Elsevier, 1986, pp. 369-419.

[32] B. C. Moore, "Principal component analysis in linear systems: controllability, observability and model reduction," IEEE Trans. Automat. Contr., vol. AC-26, pp. 17-32, Jan. 1981.

[33] M. Morari, "Design of resilient processing plants III. A general framework for the assessment of dynamic resilience," Chem. Eng. Sci., vol. 38, no. 11, pp. 1881-1891, 1983.

[34] M. Morari, E. Zafiriou, and B. R. Holt, "Design of resilient processing plants-X. New characterization of the effect of RHP zeros," Chem. Eng. Sci., vol. 42, no. 10, pp. 2425-2428, 1987.

[35] M. Morari and E. Zafiriou, Robust Process Control. New York: Prentice-Hall, 1989

[36] K. R. Muske and J. B. Rawlings, "Model predictive control with linear models," AIChE J., vol. 39, no. 2, pp. 262-287, 1993.

[37] G. de Nicolao and S. Strada, "On the stability of receding horizon LQ with zero-state terminal constraint," IEEE Trans. Automat. Contr., vol. 22, pp. 257-260, Feb. 1997.

[38] R. J. Ober and J. A. Sefton, "Stability of control systems and graphs of linear systems," Syst. Control Lett., vol. 17, pp. 265-280, 1991.

[39] M. A. Poubelle, R. R. Bitmead, and M. R. Gevers, "Fake algebraic Riccati techniques and stability," IEEE Trans. Automat. Contr., vol. 33, pp. 379-381, Mar. 1988.

[40] J. B. Rawlings and K. R. Muske, "The stability of constrained receding horizon control," IEEE Trans. Automat. Contr., vol. 38, pp. 1512-1516, Oct. 1993.

[41] M. Rosenblum and J. Rovnyak, Hardy Spaces and Operator Theory. New York: Oxford Univ.Press, 1985.

[42] H. H. Rosenbrock, State Space and Multivariable Theory. New York: Academic, 1970.

[43] L. W. Russel and J. D. Perkins, "Toward a method for diagnosis of controllability and operability problems in chemical plants," Chem. Eng. Res. Des., vol. 65, pp. 453-461, 1987.

[44] R. Scattolini and S. Bittanti, "On the choice of the horizon in long range predictive control-Some simple criteria," Automatica, vol. 26, no. 5, pp. 915-917, 1990.

[45] S. Skogestad, M. Hovd, and P. Lundström, "Simple frequency domain tools for analysis of inherent control limitations," Model., Ident., Control, vol. 12, no. 4, pp. 159-177, 1991.

[46] S. Skogestad and I. Postlethwaite, Multivariable Feedback Control, Analysis and Design. Chichester, U.K.: Wiley, 1996.

[47] A. Stoorvogel and J. Ludlage, "Squaring-down and the problems of almost-zeros for continuous time systems," Syst. Control Lett., vol. 23, no. 5, pp. 381-388, 1994.

[48] A. Stoorvogel, S. Weiland, and J. Ludlage, "On asymptotic stability of a receding horizon control scheme," in Proc. IEEE Conf. Decision Control, Phoenix, AZ, 1999, pp. 3684-3689.

[49] M. Sznaier and M. J. Damborg, "Heuristically enhanced feedback control of constrained discrete time linear systems," Automatica, vol. 6, no. 3, pp. 521-532, 1990. 
[50] M. C. Tsai, D. W. Gu, I. Postlethwaite, and B. D. O. Anderson, "Inner functions and a pseudo singular value decomposition in super optimal $H_{\infty}$ control," Int. J. Control, vol. 51, no. 5, pp. 1119-1131, 1990.

[51] C. A. Tsiligiannis and S. A. Svoronos, "Dynamic interactor in multivariable process control-I. The general time delay case," Chem. Eng. Sci., vol. 43 , no. 2 , pp. $339-347,1988$

[52] — - "Dynamic interactor in multivariable process control-II. Time delays and zeros outside the unit circle," Chem. Eng. Sci., vol. 44, no. 9, pp. 2041-2047, 1989.

[53] M. Vidyasagar, Control System Synthesis. A Factorization Approach. Cambridge, MA: MIT Press, 1985.

[54] S. Weiland, A. A. Stoorvogel, and A. Tiagounov, "End-point parametrization and guaranteed stability for a model predictive control scheme," in Proc. IEEE Conf. Decision Control, Orlando, FL, 2001, pp. 4832-4837.

[55] W. A. Wolovich and P. L. Falb, "Invariants and canonical forms under dynamic compensation," SIAM J. Control Optim., vol. 14, no. 6, pp. 906-1008, 1976.

[56] J. G. Ziegler and N. B. Nichols, "Process lags in automatic circuits," Trans. ASME, vol. 65, pp. 433-444, 1943.

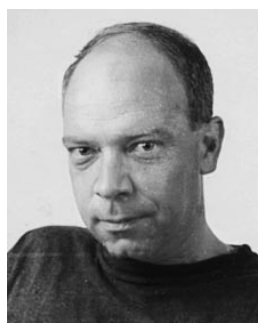

Jobert H. A. Ludlage (M'93) received the M.Sc. degree in electrical engineering and the Ph.D. degree on the subject of controllability analysis for industrial processes, both from Eindhoven University of Technology, Eindhoven, The Netherlands, in 1987 and 1997, respectively

Since 1987, he has been working as a process control engineer in process industry. Since 1998, he has been with IPCOS Technology, The Netherlands, where he is responsible for the development of the INCA control technology. As such, he is involved in a number of international research and development projects. His R\&D interests are in the field of identification, real-time optimization, and model predictive control and their application in process industry. His current activities are focused on the use of detailed rigorous dynamic models in different layers of the process control hierarchy.

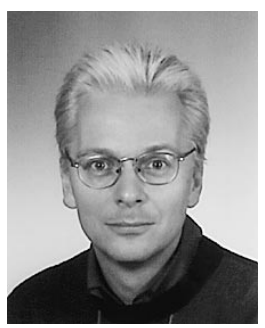

Siep Weiland received the M.Sc. and Ph.D. degrees in mathematics and physical sciences from Groningen University, Groningen, The Netherlands, in 1986 and 1991, respectively.

From 1991 to 1992, he has held a Postdoctoral Research position at the Department of Electrical and Computer Engineering at Rice University, Houston, TX. In 1992, he joined the Department of Electrical Engineering at Eindhoven University of Technology, Eindhoven, The Netherlands, where he is currently Associate Professor in systems and control. His research interests include general systems theory, robust control, and model approximation.

Dr. Weiland has been Associate Editor of the IEEE TRANSACTIONS ON AUTOMATIC CONTROL, and is currently Associate Editor of the International Journal of Nonlinear and Robust Control, the European Journal of Control, and Automatica.

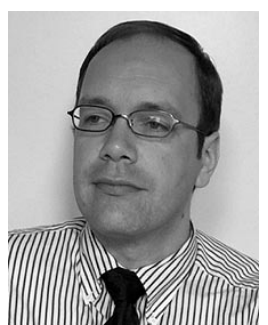

Anton A. Stoorvogel (M'91-SM'00) received the M.Sc. degree in mathematics from Leiden University, Leiden, The Netherlands, and the Ph.D. degree in mathematics from Eindhoven University of Technology, Eindhoven, The Netherlands, in 1987 and 1990, respectively.

$\mathrm{He}$ has been associated with Eindhoven University of Technology since 1987 . In 2000 , he was appointed Professor in the Department of Information Technology and Systems of Delft University of Technology, Delft, The Netherlands, and as Professor and Adjunct Faculty Member in the Department of Electrical Engineering and Computer Science of Washington State University, Pullman. In 1991, he visited the University of Michigan, Ann Arbor. From 1991 to 1996, he was a Researcher of the Royal Netherlands Academy of Sciences, The Netherlands. He is the author of three books and numerous articles.

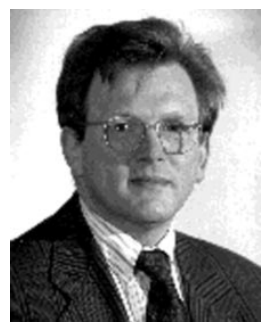

Ton A. C. P. M. Backx received the M.Sc. degree in electrical engineering in 1976 and the Ph.D. degree, from Eindhoven University of Technology, Eindhoven, The Netherlands, in 1976 and 1987, respectively. His Ph.D. work was in the area of reliable and robust multi-input-multi-output process indentification methods.

$\mathrm{He}$ is currently president of IPCOS Technology, The Netherlands, a high-tech company supplying state-of-the-art model predictive control and dynamic optimization technology. Besides technology licenses, the company also supplies consultancy and engineering services, and executes projects in the area of MPC and dynamic real-time optimization. He has been and still is involved in consultancy and the realization of advanced control and optimization projects in hydrocarbon processing, chemical processing, polymer production, lamp manufacturing, and glass manufacturing industry. He has been a part-time Professor at the Technical University of Eindhoven, Eindhoven, The Netherlands, since 1990. He is Technical Coordinator of several European research projects (IMPACT, INCOOP, SINC-PRO, and MATCH). His research interests are in the field of high-performance (non-)linear MPC, state estimation, and dynamic real-time optimization. 